\title{
Load Feasible Region Determination by Using Adaptive Particle Swarm Optimization
}

\author{
Patchrapa Wongchai ${ }^{\mathrm{a}}$ and Sotdhipong Phichaisawat ${ }^{\mathrm{b},}$ \\ Department of Electrical Engineering, Faculty of Engineering, Chulalongkorn University, Bangkok 10330, \\ Thailand \\ E-mail: apatchrapa.w@student.chula.ac.th, bsotdhipong.p@Chula.ac.th
}

\begin{abstract}
The proposed method determines points in a feasible region by using an adaptive particle swarm optimization in order to solve the boundary region which represented by the obtained points. This method is also used for calculating a large-scale power system. In any contingency case, it will be illustrated with an $\mathrm{x}$-axis and $\mathrm{y}$-axis space which is given by the power flow analysis. In addition, this presented approach in this paper not only demonstrates the optimal points through the boundary tracing method of the feasible region but also presents the boundary points obtained the particle swarm optimization. Moreover, decreasing loss function and operational physical constraints such as voltage level, equipment specification are all simultaneously considered. The points in the feasible region are also determined the boundary points which a point happening a contingency in the power system is already taken into account and the stability of load demand is ascertained into the normal operation, i.e. the power system can be run without violation. These feasible points regulate the actions of the system and the robustness of the operating points. Finally, the proposed method is evaluated on the test system to examine the impact of system parameters relevant to generation and consumption.
\end{abstract}

Keywords: Particle swarm optimization, feasible region, boundary tracing method, power flow solution space.

ENGINEERING JOURNAL Volume 23 Issue 6

Received 28 July 2019

Accepted 30 September 2019

Published 30 November 2019

Online at http://www.engj.org/

DOI:10.4186/ej.2019.23.6.239 


\section{Introduction}

The electrical power system analysis has a wide range of conditions for determining problems, consisting of three main systems: generation system, transmission system, and distribution system. A major system that is analyzed in practice is the transmission system with two main components. The electrical power supplies to users in both primary and secondary feeders are mostly used to solve these transmission problems by taking into account the power loss and voltage drop in the power flow equations. A regularly occurring barrier is the violation of bus voltage and overload of transmission lines. In addition, the increase of loads can also be limited by the critical point, where the critical point must be stabilized by the voltage level. This is referred to as the steady-state voltage stability analysis [1],[2]. Therefore, the load-point configuration that supports the power system can normally operate. The receiving load margin must be carefully considered and analyzed by considering an available transfer capability (ATC). The apparent power is a two-part power output consisting of the real and reactive powers that are used to consider the ATC and the use of theoretical calculation powers through large power systems [3],[4].

As aforementioned, the load margin that the system can be accepted is a measure of the ability to support the increased load, which is determined the load point where the supported system can be obtained from several different theories such as repeated power flow (RPF), continuation power flow (CPF), optimal power flow (OPF) [5]-[7]. The fundamental methods in the operation of active and reactive power control (Walt/VAr control) solutions relate to many operations to determine the supported load-point boundary. This is the best way to find the combinatorial optimization problem method. The ATC is accurate and takes less computation time when the number of buses is not too large but if it is a very large power system consisting of a very large number of buses, it is necessary to find the appropriate method for calculations. Until now, there are systematic approaches to find the most common value such as conventional optimization algorithms to avoid the error therefore it is possible to find the most appropriate method for the particle group method. The particle swarm optimization (PSO) [8] and the TNEP model [9] are applied in conjunction with a certain scope point to find the feasible region.

Due to the uncertainty of a power system, if power of the electrical system is discharged through a transmission line, it is still possible to maintain stable operation conditions and to meet complex conditions. It is usually fast to find out the range of spatial answers that bring to a good answer [10]. A variety of feasible region points supported by the system can be described through the pattern, stabilization, scope of system operation and transmission in each bus. The optimization of a set variables is a sequential point, each of which shows the balance of a support load boundary that has been shown in the form of multiple dots. This research aims to develop a tracing boundary method of determining the security boundaries of the system under the conditions presented by adaptive particle swarm optimization (APSO), where it is both impossible and possible areas of the combinations being called solvable region [10]-[14]. The proposed method is used to determine the boundary tracing as the basis of the subject process. The predictor and corrector methods are used to find the right boundary tracing known as continuation method [14]. The predicted point is the precautionary boundary extent that the value is located. The power flow is a singular Jacobian matrix [16][18], where the precautionary boundary can be met according to a linear equation that consists of power flow equation. It can be applied to the load feasible region process to determine the spot area. However, the load feasible region in [15] and [19] is not focused only on the scope to determine the specific operation points but also security area assignments are applied for transmission line between the source-load pair in power systems.

This paper is divided into four sections consisting of (1) solvable region of power flow solution, (2) problem description, (3) numerical results, and (4) conclusions. In the first section, the electrical analysis problem with the origin of the feasible region is the method used in this research consisting of feasible region, OPF method and solvable region of boundary points, and boundary tracing method with solvable region. The second section discusses the previous problems and obligations with particle swarm that is applied in this research. This state consists of decision variables, state formulation of problem, adaptive PSO method, replacement the problem with particle swarm method, and computation procedure. The numerical results in the third section are demonstrated by comparing the experimental results of the previous methods and the proposed adaptive particle swarm method. Finally, it ends with the conclusions. 


\section{Solvable Region of Power Flow Solution}

\subsection{Feasible Region}

The feasible region is consideration to be divided into three periods, where the region obtained by power flow solution that is the solvable boundary $\left(\Sigma_{1}\right)$ and the feasible boundary $\left(\Sigma_{2}\right)$ [7], defined in the figure below. The space where the computational has no answer is call "the unsolvable boundary". The infeasible boundary is the subset of solvable points, but one of these points exceed execution limit. On other hand, the feasible region is the space where all of system parameters are execute condition their limit.

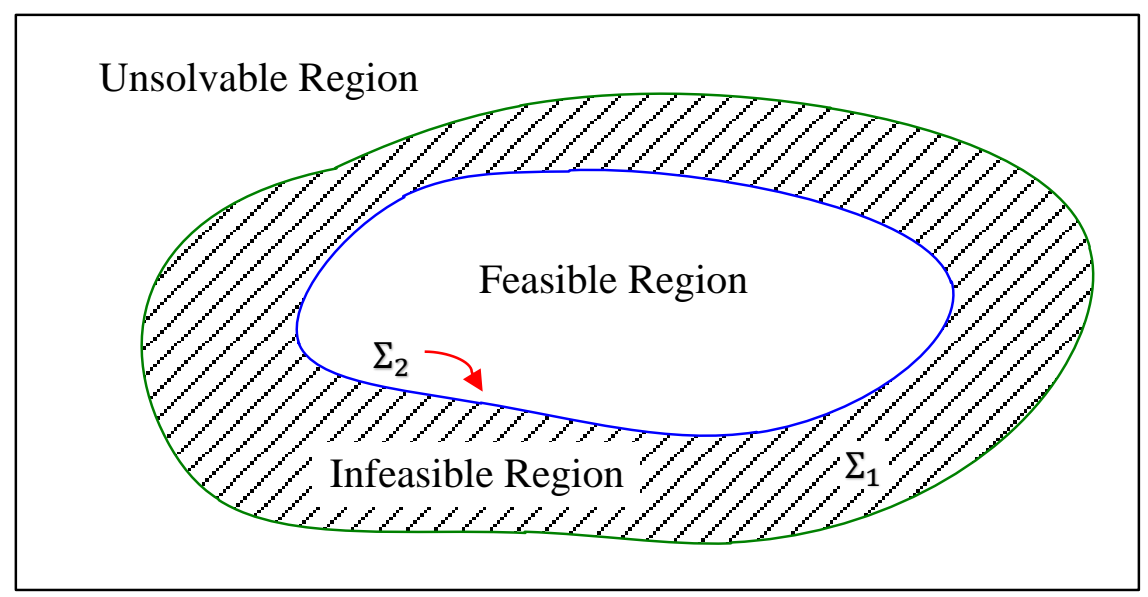

Fig. 1. The area of operating points [20].

\subsection{OPF Method and Solvable Region of Boundary Points}

The region boundary is a space within solvable region which represent the critical points and defined SNB [20]. The SNB point obtained by the power flow solution that is corresponding with a singular jacobian matrix and maximum load parameter. The power flow solution inoperative, when the load demand exceeding the SNB point due to varies of the load direction increment and the load change in parameter of power flow solution. In addition, the SNB point is the minimization to solve the result from the unsolvable area. The determination to SNB point can be classify into two method from external the solvable boundary [11]-[15].

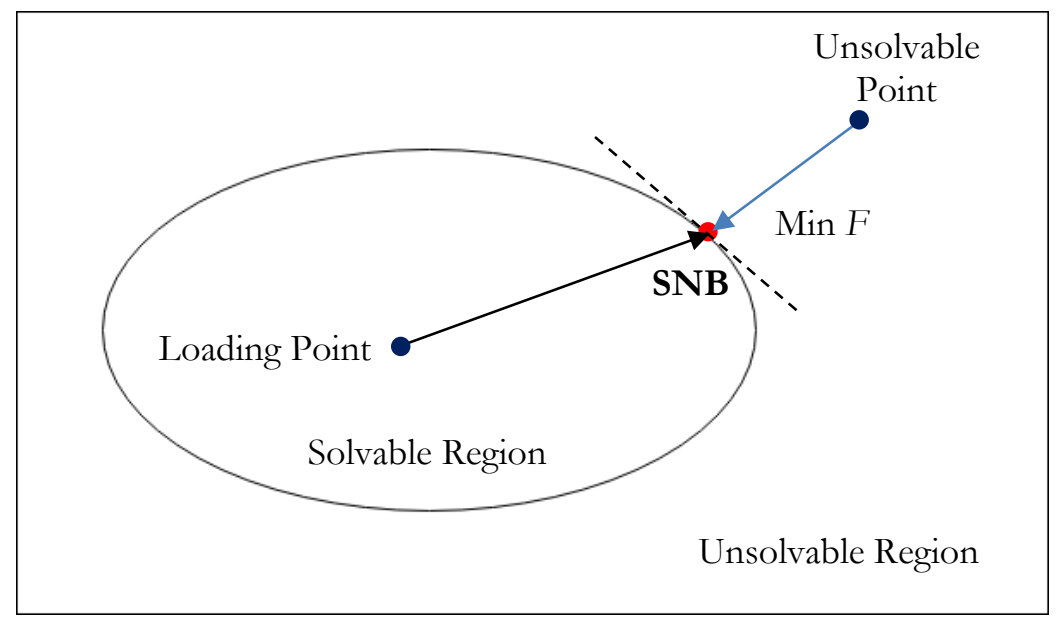

Fig. 2. Demonstration of optimization methods.

The maximization from within the solvable boundary is the computational to solve the load variable subject to optimal power flow algebraic constrains as equations: 


$$
\text { s.t. } g(x, \lambda)=0
$$

The solution point obtain within the maximum solvable region is the SNB point which is equilibrium the CPF method.

The computational method from external the feasible region obtained by minimize the Euclidean space from the unsolvable region [7]. From the external region solvable bounadary $\left(S^{*}\right)$, the nearest solvable region determined by the unconstrained minimization:

$$
\min F(x)=\frac{1}{2}\left[f(x)-S^{*}\right]^{T}\left[f(x)-S^{*}\right]
$$

where $S^{*}$

is a injection matrix from unsolvable points;

$$
\begin{array}{ll}
F(x): \mathbb{R}^{N} \rightarrow \mathbb{R} & \begin{array}{l}
\text { represent a cost function, obtained by one and half of the square power flow } \\
\text { mismatch. }
\end{array}
\end{array}
$$

The formulation result shown the SNB point from the unsolvable boundary represented by $S^{*}$. The power injections represented by $S$ can formulate from vector $x$ and produces the vector $S-S^{*}$ parallel with the regular vector for trace boundary of solvable boundary from the point $S$. This method is beneficial for the stability balance in order to solve the corrective process for control action from the external unsolvable boundary.

\subsection{Boundary Tracing Method with Solvable Region}

The boundary region is the curve that categorized the solution space of optimal power flow into two areas are unsolvable region and solvable region. The Jacobian matrix is a singular matrix that corresponding with the boundary point. In addition, the predictor-corrector process use to determination the boundary region of solvable region by the tracing boundary algorithm. The corrector procedure use to optimization problem by minimize point from previous distance. The prediction procedure is forward to develop in order to certify the solution point from predictor within the feasible boundary. The first prediction point represented by $S_{1}^{*}$ it is the initial tracing procedure and $X^{0}$ is represent the first initial guess obtained by formulation the repeated power flow method.

The determination of tracing boundary process needs to defined the first prediction point is $S^{*}$ and the first initial guess is $X^{0}$ to the parameters problem. The variable $S^{*}$ is a predictor term to predict the loading point for ensure all of parameters not violation in the test system. The first initial guess $X^{0}$ obtained by optimal power flow should be nearest the boundary curve. The repeated power flow uses to determine the system parameter, which the concept is increasing of the loading point at the load bus each step represent by $\sigma$ per MVA. In addition, the variable $S^{b}$ of tracing boundary method is search the point by the bisection method. The fist point after formulation obtained infeasible point is $S^{*}$ for determination increasing load point from $S^{b}$ applying the repeated power flow with the corresponding direction $\theta$. The processing explained in Fig. 3. 


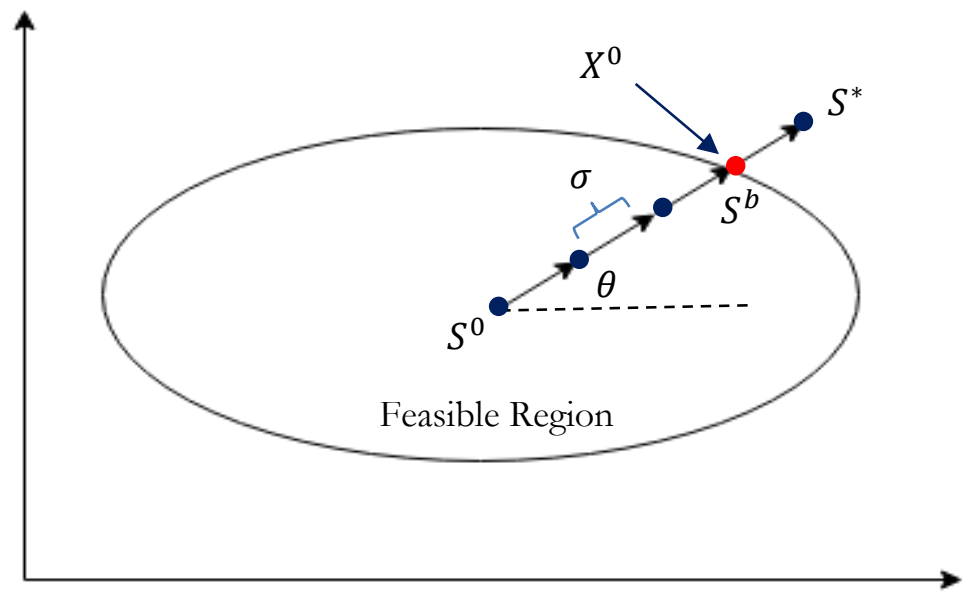

Fig. 3. Optimization to determine initial infeasible point.

\subsubsection{Process of correction}

The process of correction to minimize the problem as explained in [22]. The inputs of this manipulation to predict the point $S^{*}$ and the first prediction $X^{0}$. The performance of function $X$ to check the result of minimization problem that is the point $S^{*}$ within the feasible boundary or not. The result can be classified into two problems:

- The problem of $F(X)=0$ implicate the predicted point $S^{*}$ is internal the feasible boundary, and the result of function $X$ is not the answer on the boundary point.

- The problem of $F(X)>0$ implicate the prediction point $S^{*}$ is undisturbed external the feasible boundary, and the final result $X$ is the answer on the boundary point.

If the procedure of prediction point $S^{*}$ is internal the feasible region, the procedure satisfied by determine a new predicted point by the step in [22].

\subsubsection{Process of prediction}

The process of prediction is to guess the forward calculation of boundary region, which can be calculated by application the previous solution point, and the space between the predicted point and the graph of boundary point can be improved. Let $d$ represent the length of variable for modification the space by the tracing process $k$, the point $S_{k}^{* \prime}$ is explained in [22].

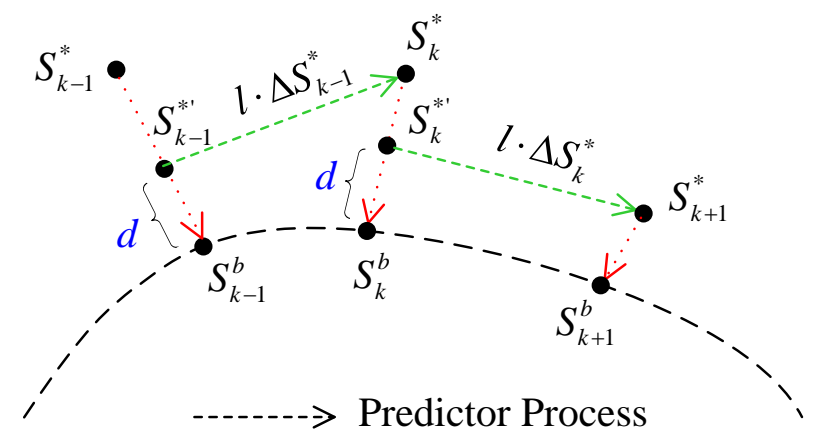

Fig. 4. Predictor process with space adjustment. 


\section{Problem Description}

The transmission system has several performance considerations: reducing the power loss of system, adjusting the voltage at each bus appropriate for the precautionary boundary and the power quality. The above requirements allow a power system to support loads that will be added in the future. However, the above mentioned, benefits will be occurred when the performance is subject to acceptable system conditions. Therefore, the installation of the transmission lines with contingencies from the system will be taken into account in order to get the feasible region, leading to difficulties to evaluate. Especially, in the distribution system, there are several ways to consider such as format arrangement, load distribution, and load variation that is changed throughout the days. The contingency must be carried out on the distribution system that is considered so that the system still maintains the stability of the operation and the maximum benefit. It is involved in planning to determine the appropriate contingency patterns in terms of the line position (system bus number), size (real power and reactive power at each bus), and the total number of transmission lines that remain active on the entire system on duration time.

\subsection{Decision Variables}

In this case study, there are three different types of contingency issues presented by [24] consist of: Type $A$, Type $B$, and Type $C$. In the variable decisions of each issue, $N C$ is a set consisting of bus numbers for considering the contingency. The basic information from the NC set is the format of the issue. The following is the details of each issue:

Problem statement of Type A: the information known by the NC set is the bus numbers. It means that both the number of buses and the bus positions occurred contingency will already be set. The problem statement is to find real power and reactive power at each bus with various load levels: they are all the decision of the variables. Therefore, in this issue, it will be considered only the real power and reactive power at each bus.

Problem statement of Type B: the information known by the $N C$ set is the number of buses, but it has not known what each member is defined (unknown position) yet. The problem statement is to determine how many buses needed to be occurred contingency (lost energy), the number of buses and bus positions where the contingency is occurred, and the power for each remaining bus at each load level. These variables are determined in this problem including the bus number and power (both real and reactive power) at each bus.

Problem statement of Type $C$ : the NC set does not contain any information about the contingency of the transmission line. Consequently, the problem cannot be determined any conditions of the contingency in the transmission line. The problem statement is to determine how many lines rejected when the contingency occurred, bus position, and power at each remaining bus at different load levels. In this step, the variable decision of the problem consists of the number of buses, bus position, and power at each bus.

\subsection{State Formulation of the Problem}

The objective function of the simulation of the contingency in transmission line has to be considered the control of the bus voltage in the acceptable range and the system security is a significant problem. The adaptive model of TNEP [9] is considered in any problem in case of contingencies. The analysis of case contingency must be represented by $N-1$ denote the state of power system after the transmission lines outage. The TNEP model considered with security limits, the equation of computational can be summarized as follows:

$$
\max v=\sum_{l \in \Omega} P_{l} n_{l}
$$

s.t.

$$
S f^{k}+g=d
$$




$$
\begin{gathered}
f_{l}^{k}-\gamma_{l}\left(n_{l}^{0}+n_{l}\right)\left(\Delta \theta_{l}^{k}\right)=0, \text { for } l \in 1,2 \ldots, n l \& l \neq k \\
f_{l}^{k}-\gamma_{l}\left(n_{l}^{0}+n_{l}-l\right)\left(\Delta \theta_{l}^{k}\right)=0, \text { for } l=k \\
\left|f_{l}^{k}\right| \leq\left(n_{l}^{0}+n_{l}\right) \bar{f}_{l}, \text { for } l \in 1,2, \ldots, n l \& l \neq k \\
\left|f_{l}^{k}\right| \leq\left(n_{l}^{0}+n_{l}-1\right) \bar{f}_{l}, \text { for } l=k \\
0 \leq n_{l} \leq \bar{n}_{l} \\
n_{l} \leq 0, \text { for } l \in 1,2, \ldots, n l \& l \neq k,\left(n_{l}+n_{l}^{0}-1\right) \geq 0, \text { for } l \in k, \Omega \text { and } k=0,1
\end{gathered}
$$

where $\quad k=0 \quad$ is the foundation case not have any line outage.

$S \quad$ is the node of each branch incidence matrix transposed in the line system.

$f^{k} \quad$ is the vector of $f_{l}^{k}$.

$\gamma_{l} \quad$ is the circuit susceptive addition to $l^{\text {th }}$.

$n_{l} \quad$ is the circuit number addition to $l^{\text {th }}$.

$n_{l}^{0} \quad$ is the zero circuit in normal operation.

$\Delta \theta_{l}^{k} \quad$ is the step phase angle when $k^{\text {th }}$ is the number of line contingencies.

$f_{l}^{k} \quad$ is the summarized of active power obtained by the circuit of $l^{\text {th }}$.

$\bar{f} \quad$ is the maximum operation point of active power obtained by the circuit of $l^{\text {th }}$.

$\bar{n}_{l} \quad$ is the maximum number of line circuit that can addition in $l^{\text {th }}$.

$\Omega \quad$ is the set of all transmission lines.

$P_{l} \quad$ is the total of active power of all transmission lines.

$n_{l} \quad$ is the total number of all transmission lines.

$N C \quad$ is the number of probability contingencies.

The objective function of the problem is to maximize the total active power in all of transmission lines existed after the line contingencies occur in the power system, satisfying the constraints of active power flow in the lines transmission system, for the fundamental case of contingency defined by $N-1$. (5) is the power balance, (6) and (7) are the power flow equation of active power. (8) and (9) the constraints of active power flow, and (10) presents the line constraints.

\subsection{Adaptive PSO Method}

\subsubsection{Fundamental of PSO}

The PSO was first established by [25]. The execution of PSO including an inertial weight and a constriction factor. All review detail of development in PSO is presented in [25]-[27]. The PSO method is the search tool using by population of particle and corresponding to each self-particle. The PSO presents competitor solution to solve the problem. The first step is a random initialization of population in each search space and executes on the particles social for observation behavior in the swarm such as fish, bee, bird, and the swarm theory. The objective to finds the global optimal by simply changing the trajectory of individual in each the search space that is changed by dynamically modifying the velocity of each particle, learning to the flying experience itself and the other particles in the search own area. 


\subsection{2. $\mathrm{PSO}$ and comparison of advantages}

1) The PSO is simplify to achieve operation and have a little process to adjust parameters. Unlike GA has development step performance such as mutation and crossover [21].

2) The GA algorithm active by sharing information, consequently the whole population of GA will be move as one direction in the group, but The PSO only the best particle $\left(\mathrm{G}_{\text {best }}\right)$ will provides the information to another particle in the swarm. It is robustness point of PSO more than GA.

3) The PSO has performance index or objective function to conduct the direction of search space problem.

4) The PSO has flexible process to control parameters between the global and local for exploration of the search space. Different the GA and other algorithms this support PSO forward to the unique feature exceeds the other heuristic tools and improve the search ability.

\subsubsection{The algorithm of PSO}

The positions and the velocities using in the initial particles swarm both of them processing by randomly produced to permit all particles randomly circulated pass over the search space. The parameter of fitness value to determine each particle for estimation, after that the particles will be determine the best position of each particle and also shown the parameter of particle that is the best value of global fitness in the present iteration. The next step of velocities in all particles are developed by using the data from the last velocity. The syntax of $G_{\text {best }}$ represents the best solution of each particle, that obtained by the best particle in swarm. The velocity has updated by development in each iterative countering $t$ to $t+1$ can be explained as [28]:

$$
v_{i d}(t+1)=w v_{i d}(t)+c_{1} r_{1 d}(t)\left[y_{i d}(t)-x_{i d}(t)\right]+c_{2} r_{2 d}(t)\left[\hat{y}_{d}(t)-x_{i d}(t)\right]
$$

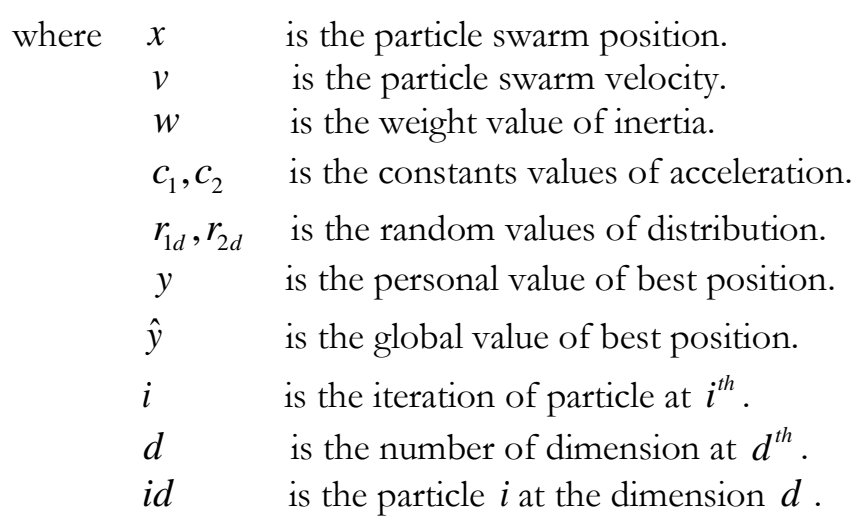

When the last position finished will be update the new position in each particle of swarm is performed by:

$$
x_{i d}(t+1)=x_{i d}(t)+v_{i d}(t+1)
$$

The procedure of velocity and position development is repeated and will be finished when the alleviation critical is encounter, for example the maximum value of iteration is terminated or the solution is adaptive smaller than the sufferance value of the pre-specified.

\subsubsection{The algorithm of adaptive PSO}

The fundamental of PSO method has the initial weight for stimulation constant of all particle in the first generation. The important parameter will be move from the state of current position to the new position for optimization by using initial weight $(w)$. In order to develop the ability of search space and the algorithm of adaptive PSO can be redeemed in the feature of particle movement, that controlled by the objective function. The adaptive PSO has a process for adjust position such that specific fitted particle which is best particle. The best particle will be more slowly than normal particle when compared each other. The procedure of 
adaptive particle can be completed be chosen different parameters in each particle swarm depend on their rank, that compare between $w_{\min }$ and $w_{\max }$ as explained following [9]:

$$
w_{1}=w_{\text {min }}+\frac{\left(w_{\text {max }}-w_{\text {min }}\right) * \operatorname{Rank}_{i}}{\text { Total population }}
$$

Therefore, from (13) can be concluded the best particle is the best of first rank and inertia weight for determination the maximum value and the lowest fitted particle will represents the maximum of inertia weight, consequently the particle will move with a high velocity. The particle can be set the velocities to update and develop behavior by using (14), and if the parameters of velocity value exceeds than $V_{\max }$, it can be ensured by (15).

$$
\begin{gathered}
v_{i j}(t+1)=w_{i} v_{i j}(t)+c_{1} r_{1}\left(p_{i j}(t)-x_{i j}(t)\right)+c_{2} r_{2}\left(p_{g j}(t)-x_{i j}(t)\right) \\
v_{i j}(t+1)=\operatorname{sign}\left(v_{i j}(t+1)\right) * \min \left(\left|v_{i j}(t+1)\right|, V_{j \max }\right) \\
\text { for } j=1,2, \ldots, d ; i=1,2, \ldots, n
\end{gathered}
$$

The update position of particle swarm can be obtained by using (16) and the value of particles position exceed the determination range, then it can be adjusted to feasible region by (17).

$$
\begin{aligned}
x_{i j}(t+1)= & x_{i j}(t)+v_{i j}(t+1), \text { for } j=1,2, \ldots, d ; i=1,2, \ldots, n \\
& x_{i j}(t+1)=\min \left(x_{i j}(t+1), \text { range }_{j \max }\right) \\
& x_{i j}(t+1)=\max \left(x_{i j}(t+1), \text { range }_{j \max }\right)
\end{aligned}
$$

The propose of re-initialization is presented to the APSO algorithm later than define the number of generations when the convergence of the algorithm is not development. The above mentioned of APSO population has a unique feature generation is re-initialized by new randomly produced individuals. The new individuals number chosen from $\mathrm{k}$ when $\mathrm{k}$ is the least fitted individuals of original population, measured by the percentage of all population to be adjusted. The effect re-initialization of population process is identical to the modification process of GA algorithm. The effect not only useful the algorithm precipitately converges to a local optimal but also re-initialization of population it can be obtained $\mathrm{F}_{\text {best }}$ value that is specific number of swarms.

\subsubsection{APSO and contingency}

The objective function $(f(x))$ for contingency represented by (4). When $x$ is the set of competitor lines which presenting a solution of contingencies. The element of $x$ denotes the correctly direction in each a competitor line construction. The range of each parameters defined by $x_{i}$ it can be ensured right-of-ways [9]. The fitness function evaluation is the function to inspect the worth case of a solution that can represented by:

$$
f=\sum_{l} P_{l} n_{l}+W_{1} \sum_{k=0}^{N C} \sum_{o l}\left(a b s\left(f_{l}^{k}\right)-\bar{f}_{l}\right)+W_{2}\left(n_{l}-\bar{n}_{l}\right)
$$

Where syntax $o l$ is the set of overloaded lines. The objective function proposed to find the set of transmission lines for formulation such that the minimization of active power must not overloads are generated during the horizontal of plan. Therefore, the first term of (18) formulations the total active power in each transmission contingencies plan. The second term is additional function for analysis the active power flow by using the constraint violations in the normal operation case, and the syntax $N-1$ is represents 
contingency cases. The third term is optimal function for find the maximum number of circuit, where the function of $l^{\text {th }}$ over the operation limits. The $W_{1}, W_{2}$ are the penalty terms. Furthermore, the second and the third terms are attached to the main objective function only case contingency of violations.

\subsection{Replacing of the PSO Method}

The objective function (18) in the application of the particle swarm method with the solution of contingency and the outage transmission lines from the system is to replace the problem with particle swarm. The variable which is executed by PSO, such as the number of variables, decisions, and load levels is taken into account. The problem is divided into the several contingency states releasing the outage line into 3 forms: Type A, Type $B$, and Type $C$. Each problem has a number of different decision variants. It is different to replace variables with the particle swarm method.

Problem statement of Type $A$ : The decision variables of the problems consist of real power and reactive power at each bus in load levels. The member of $N C$ set is the bus position number defined by $n c$, consequently the replacement with particle swarm method which is:

$$
\begin{aligned}
& \bar{x}=\left[\begin{array}{llllll}
\overline{P_{c_{1}}} & \overline{P_{c_{2}}} & \ldots & \overline{P_{c_{t}}} & \ldots & \overline{P_{c_{N C}}} \\
\overline{Q_{c_{1}}} & \overline{Q_{c_{2}}} & \ldots & \overline{Q_{c_{t}}} & \ldots & \overline{Q_{c_{N C}}}
\end{array}\right] \\
& \overline{P_{c_{t}}}=\left[\begin{array}{llllll}
P_{c_{t}^{1}} & P_{c_{t}^{2}} & \ldots & P_{c_{t}^{j}} & \ldots & P_{c_{t}^{s}}
\end{array}\right] \\
& \overline{Q_{c_{t}}}=\left[\begin{array}{llllll}
Q_{c_{t}^{1}} & Q_{c_{t}^{2}} & \ldots & Q_{c_{t}^{j}} & \ldots & Q_{c_{t}^{s}}
\end{array}\right]
\end{aligned}
$$

The number of dimensions of each particle can be calculated by:

$$
D=N C \times S
$$

Problem statement of Type B: The information from the NC set is the total number of members within the set (The number of outage line transmission). The decision variables of the problem are the bus outage number of line transmissions, and both of all power supplies are real and reactive power at each different load levels. If $n c$ is the number of in $N C$ set, each particle is represented by the following characteristics:

$$
\begin{gathered}
\bar{x}=\left[\begin{array}{lllllll}
\overline{B C} & \overline{P_{c_{1}}} & \overline{P_{c_{2}}} & \ldots & \overline{P_{c_{t}}} & \ldots & \overline{P_{c_{N C}}} \\
\overline{B C} & \overline{Q_{c_{1}}} & \overline{Q_{c_{2}}} & \ldots & \overline{Q_{c_{t}}} & \ldots & \overline{Q_{c_{N C}}}
\end{array}\right] \\
\overline{B C}=\left[\begin{array}{lllllll}
B C_{1} & B C_{2} & \ldots & B C_{t} & \ldots & B C_{N C}
\end{array}\right] \\
\overline{P_{c_{t}}}=\left[\begin{array}{lllllll}
P_{c_{t}^{1}} & P_{c_{t}^{2}} & \ldots & P_{c_{t}^{j}} & \ldots & P_{c_{t}^{s}}
\end{array}\right] \\
\overline{Q_{c_{t}}}=\left[\begin{array}{lllllll}
Q_{c_{t}^{1}} & Q_{c_{t}^{2}} & \ldots & Q_{c_{t}^{j}} & \ldots & Q_{c_{t}^{s}}
\end{array}\right]
\end{gathered}
$$

The number of dimensions of each particle can be calculated by:

$$
D=N C+(N C \times S)=N C \times(1+S)
$$


Problem statement of Type $C$ : The NC set of the problems is not defined any conditions for the transmission lines outage. The problem decision variables consist of the number of buses, bus positions and both of real power and reactive power, which are supplied to each bus at the load level. This research has applied the binary-particle swarm algorithm to reduce the number of decision variables to provide existing the bus number and the power supply demand at each bus represented by:

$$
\begin{gathered}
\bar{x}=\left[\begin{array}{lllllll}
\overline{U c} & \overline{P_{c_{1}}} & \overline{P_{c_{2}}} & \ldots & \overline{P_{c_{t}}} & \ldots & \overline{P_{c_{N B}}} \\
\overline{U c} & \overline{Q_{c_{1}}} & \overline{Q_{c_{2}}} & \ldots & \overline{Q_{c_{t}}} & \ldots & \overline{Q_{c_{N B}}}
\end{array}\right] \\
\overline{U c}=\left[\begin{array}{lllllll}
U c_{1} & U c_{2} & \ldots & U c_{k} & \ldots & U c_{N B}
\end{array}\right] ; U c_{k} \in\{0,1\} \\
\overline{P_{c_{k}}}=\left[\begin{array}{lllllll}
P_{c_{k}^{1}} & P_{c_{k}^{2}} & \ldots & P_{c_{k}^{j}} & \ldots & P_{c_{k}^{s}}
\end{array}\right] \\
\overline{Q_{c_{k}}}=\left[\begin{array}{lllllll}
Q_{c_{k}^{1}} & Q_{c_{k}^{2}} & \ldots & Q_{c_{k}^{j}} & \ldots & Q_{c_{k}^{s}}
\end{array}\right]
\end{gathered}
$$

$U c_{k}$ is a decision variable that defines transmission lines outage at each bus $(0=$ no outage, $1=$ outage). The number of dimensions of each particle can be calculated by:

$$
D=N C+(N C \times S)=N C \times(1+S)
$$

\subsection{Computation Procedure}

The computation procedure, developed on the algorithm for tracing the feasible region on the P-Q plane adaptive with PSO algorithm for optimal bus contingency within feasible region is described by the following steps:

1) Inputting line data and bus data of the system: power, voltage, system parameter, all operational constraints and PSO parameters.

2) Selecting the objective and the decision parameter for optimization computing and producing an initial quantity of particles. The data input matrix depends on replacing decision variables.

3) Determining the initial predictor point $S_{1}^{*}$ and the initial guess $X_{1}^{0}$ and letting $k=1$ for the first iteranc step.

4) Determining the upper and lower boundary of constraint conditions.

5) Determining the boundary point $S_{k}^{b}$ and the result on the region $X_{k}$ by using the minimization method.

6) Checking if the load existing point by $S_{k}^{*}$ must internal the feasible boundary or not. If $S_{k}^{*}=S_{k}^{b}$, then forward to the next step. If $S_{k}^{*} \neq S_{k}^{b}$, then forward to step 8 .

7) Determining the direction $\Delta S_{c}^{*(i)}$ by conventionalizing $w^{(i)}$ and then rotating 90 degrees counterclockwise by multiplying an imaginary axis $j$ as the following, $\Delta S_{c}^{*(i)}=j w^{(i)} /\left|w^{(i)}\right|$.

8) Setting initial iteration index when $=0$.

9) Finding the next infeasible point $S_{c}^{*(i+1)}$ followed by consideration of $\Delta S_{C}^{*(i)}$ and a step size $l$ as $S_{c}^{*(i+1)}=S_{c}^{*(i)} l \cdot \Delta S_{c}^{*(i)}$.

10) Determining the qualified and unqualified particles by ensuring bus number emerged in the initial section of all the particles.

11) Considering the qualified particles, executed the power flow computation to obtain all bus voltage consist of active and reactive power losses.

12) Checking all of constraints. If any one of all results is violated, a penalty function is done otherwise, the penalty function is zero. 
13) Evaluating of the fitness value from the qualified particles using total of active power loss and penalty function.

14) Comparison the fitness value with the best personal particle $\left(P_{\text {best }}\right)$. If the fitness result is lower than $P_{\text {best, }}$ carrying on the values of the current $P_{\text {best, }}$ and recording the particle position corresponding to parameter $P_{\text {best. }}$.

15) Selecting the minimum value of $P_{\text {best }}$ from all of the qualified particles transformed to the current global best, $G_{\text {best }}$ and recording the particle position corresponding to parameter $G_{\text {best. }}$

16) Updating the velocity and position of all the particles.

17) If the number of calculations is iterated to be maximum, the particle corresponding to the current $G_{\text {best }}$ is the optimal result and then forward to Step 12. Otherwise, set $\mathrm{t}=\mathrm{t}+1$ and return to 10.

Because of the equation constraints, the feasible region may not be satisfied at the point where two parts of different active constraints are appeared with each other. The tracing boundary method repeatedly returns the equilibrium boundary point, the new $S *$ adjusts positions around this feasible point until obtained a new region point after that PSO randomizes a point in feasible region, as shown in Fig. 5.

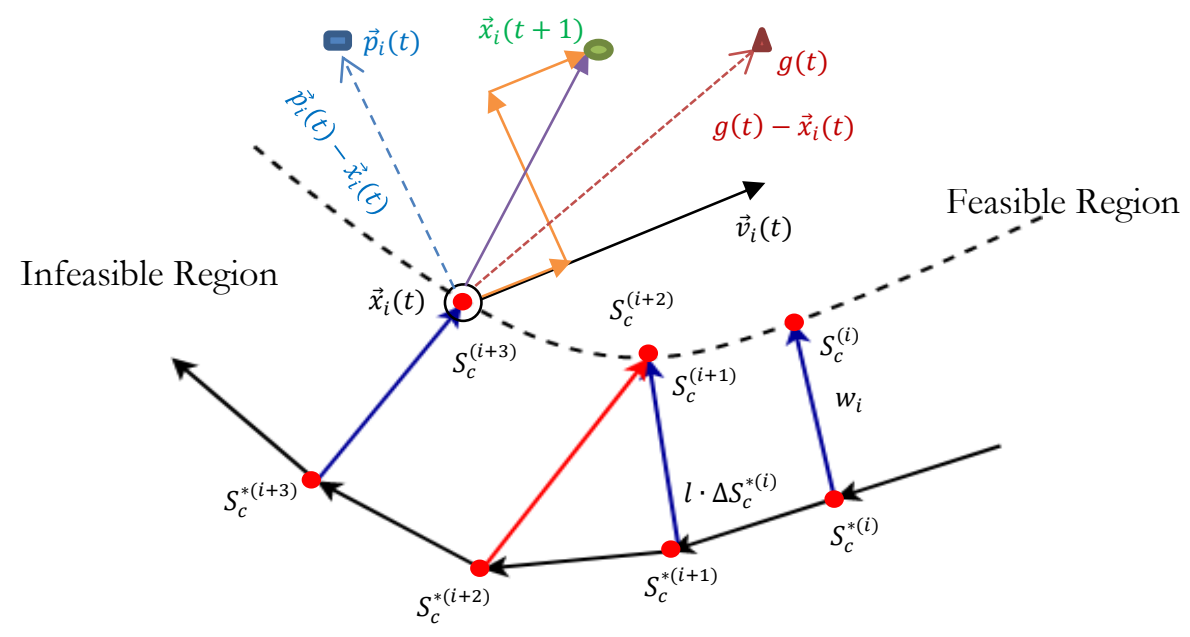

Fig. 5. Randomly PSO in Feasible boundary tracing.

\section{Numerical Results}

\subsection{Boundary Tracing Method}

The foundation of boundary tracing method is demonstrated in the two-bus system shown in Fig. 6. This demonstrates and compares the feasible boundary within the solvable boundary and then inspects the region curve obtained by determining the maximum loading points occurring in various problems.

A numerical example [15] in case of the two-bus system demonstrates the boundary within the solvable boundary by using the continuation power flow $(\mathrm{CPF})$ method. The system includes one source bus and one demand bus as displayed in Fig. 6. In this example, the magnitude of $V_{1}$ or $X$ is set to be 1.0 p.u.

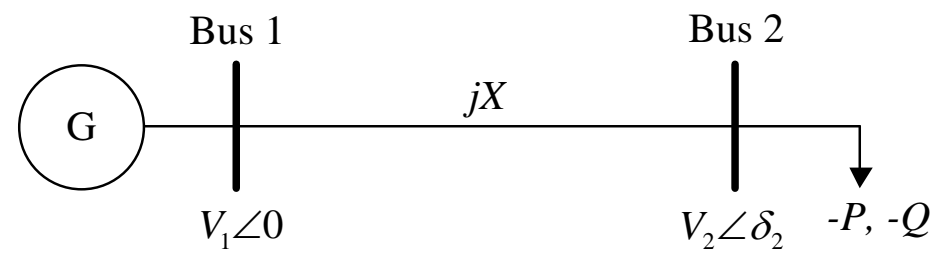

Fig. 6. A two-bus system. 
The simulation considers the demand determined by a negative value and the source is a positive value. The feasible region of boundary curve determined by the equipment's constraints of the system. The period of the voltage of bus is between 0.95 and 1.05 p.u. The period of the source capacity are between 0 and $2 \mathrm{MW}$ and \pm 2 MVAr. The demand injection is considered to be negative because of demand has no capability to generate active power. The load injection is $-0.2+j 0.0$ MVA obtained by power flow solution at Bus 2 , which is a feasible region. This point is defined to be the loading point of the computation.

From the point of existing load, the formulation returns the first point and the predictor point by application the repeated power flow method. The repeated power flow expands the demand by a step $\sigma$ of $0.1 \mathrm{MW}$ in the way of its fixed power factor. The first point is the result from the boundary at the existing loading point equal to $-0.296+j 0.0 \mathrm{MVA}$. The initial predictor dot is defined by varying the loading point of $-0.2+j 0.0 \mathrm{MVA}$. Then, the initial prediction point is $-0.496+j 0.0 \mathrm{MVA}$.

The boundary tracing method is based on the algorithm of proposed tracing boundary. The system analysis determines a step size $l$ and a space $d$ similar to 0.05 p.u. The feasible boundary contour plotted on an $x-y$ plane are active and reactive power injection at Bus 2, respectively. The boundary tracing method solution presented in Fig. 7.

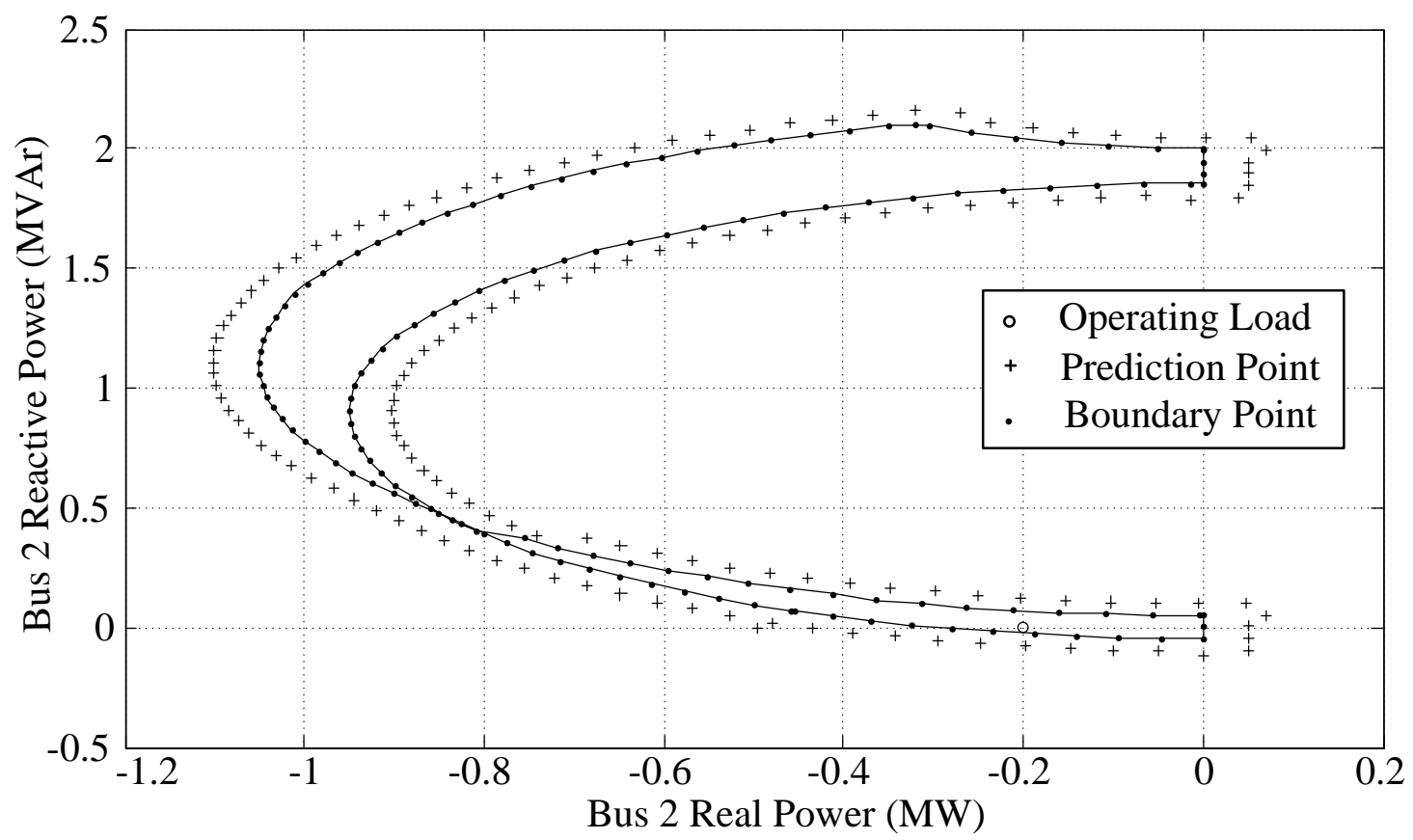

Fig. 7. Demonstration of the boundary tracing process [23].

The figure shows the loading point of existing within the boundary. In order to correlate a solution with the maximum loading points, the demand injection of $-0.8+j 1.5$ MVA is choose from fundamental of problem. From two loading points of existing, the maximum demand points are obtained by the repeated power flow algorithm. The formulation varies the load in various ways around the operating point of existing, and then correlate the solutions with the determined boundary, as presented in Fig. 8. 


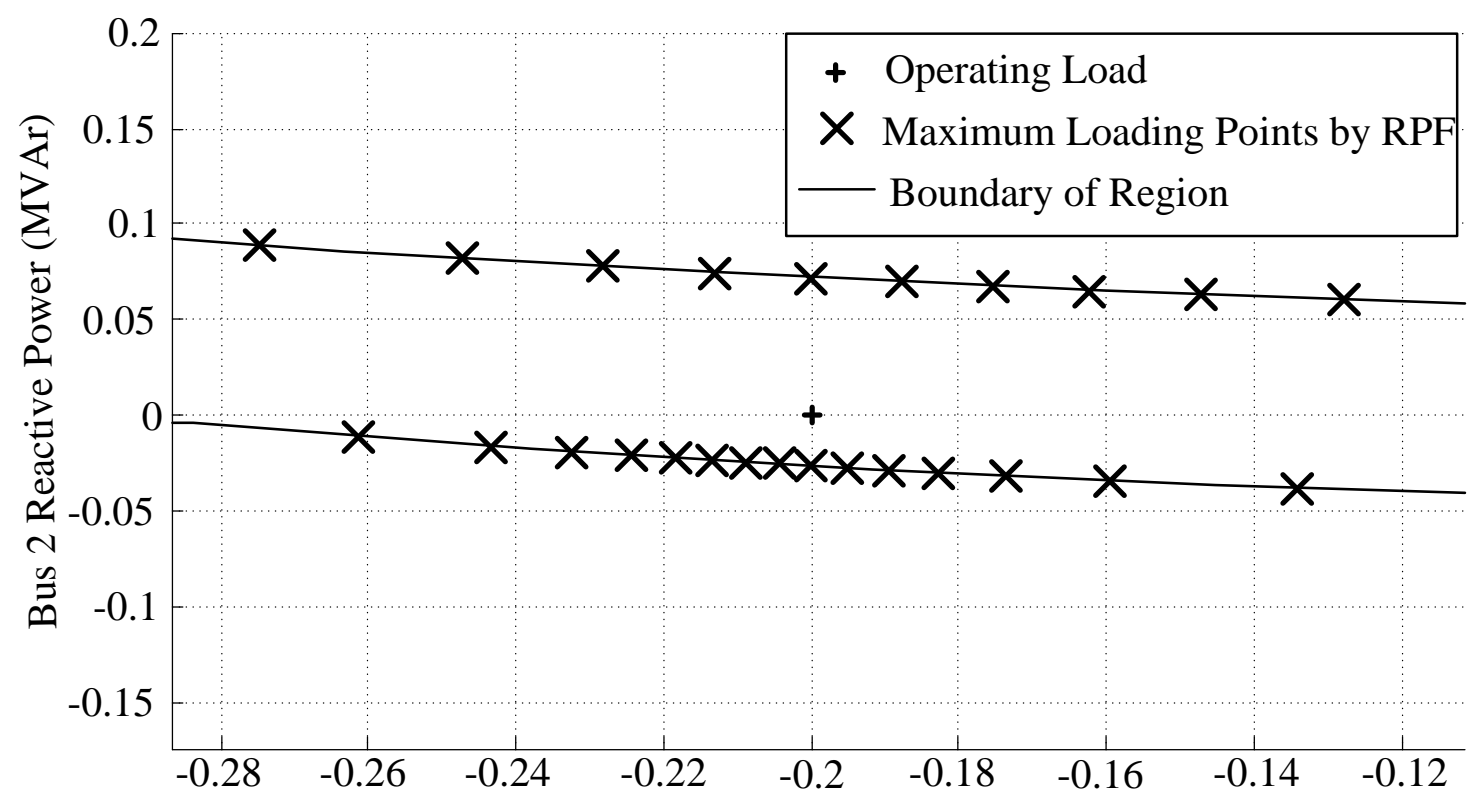

Bus 2 Real Power (MW)

Fig. 8. The Maximum loading points of $-0.2+j 0.0$ MVA [23].

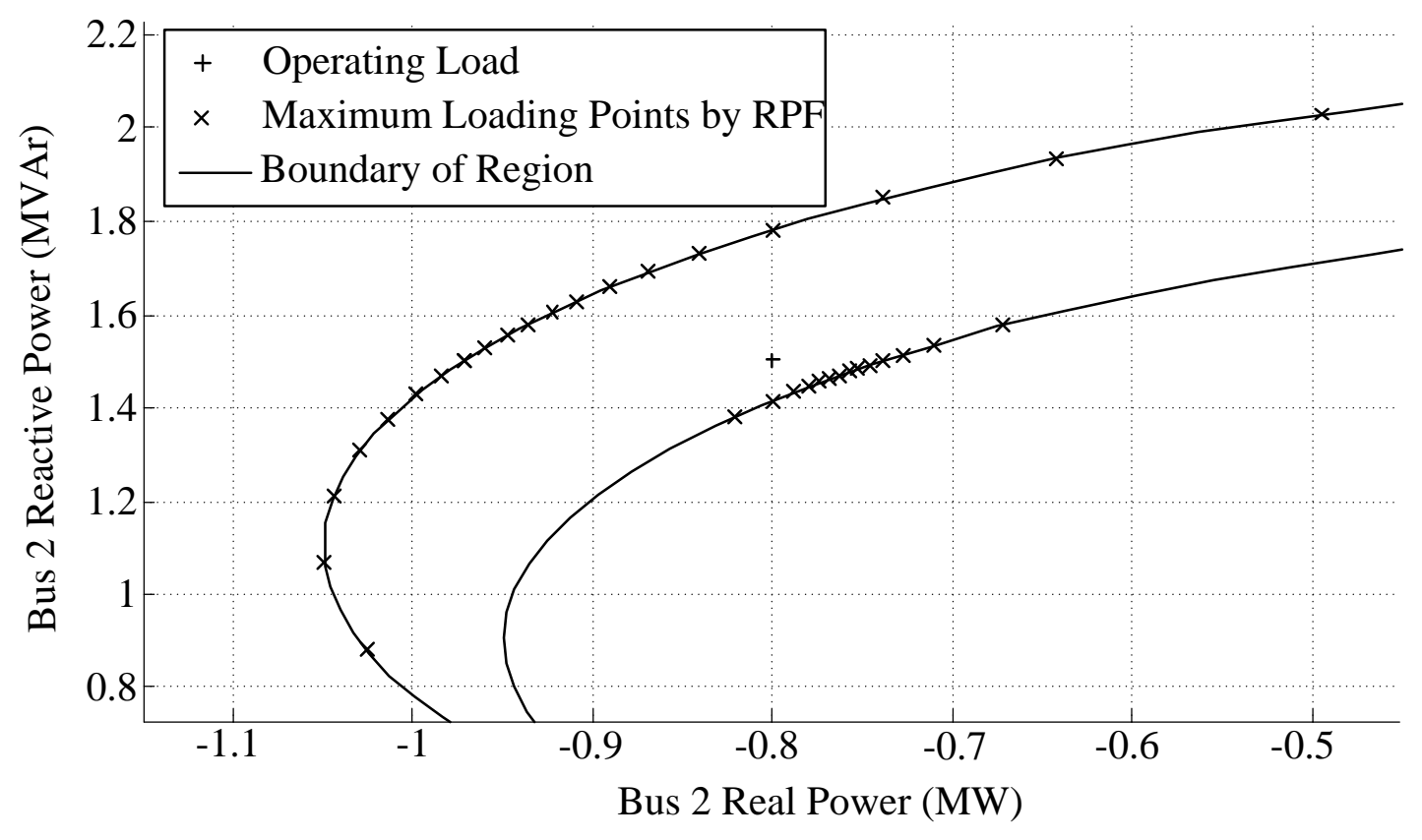

Fig. 9. The Maximum loading point of $-0.8+j 1.5$ MVA [23].

The results demonstrate the loading points obtained from maximum point stretch on the boundary curve. The boundary determined from the propess of tracing boundary method which is a curve consisting of the maximum loading points. The boundary is defined by equipment's constraints considered in this case. These constraints are practically for the power system execution. Figure 10 compares the solution of boundary with the solvable boundary. This solvable boundary is determined in [15]. A curve of solvable boundary is defined by using the process of tracing boundary method, but the minimization problem is calculated lacking equipment's constraints. From the figure, it can be complied the region bounded by the equipment's constraints is smaller than the solvable boundary. This boundary is called the feasible region as stated in several literatures [11]-[15]. However, the system analysis in this segment is a foundation problem system. 
The process of tracing boundary method can be tested on the larger power system in order to analysis the effects of the system parameters on the feasible boundary.

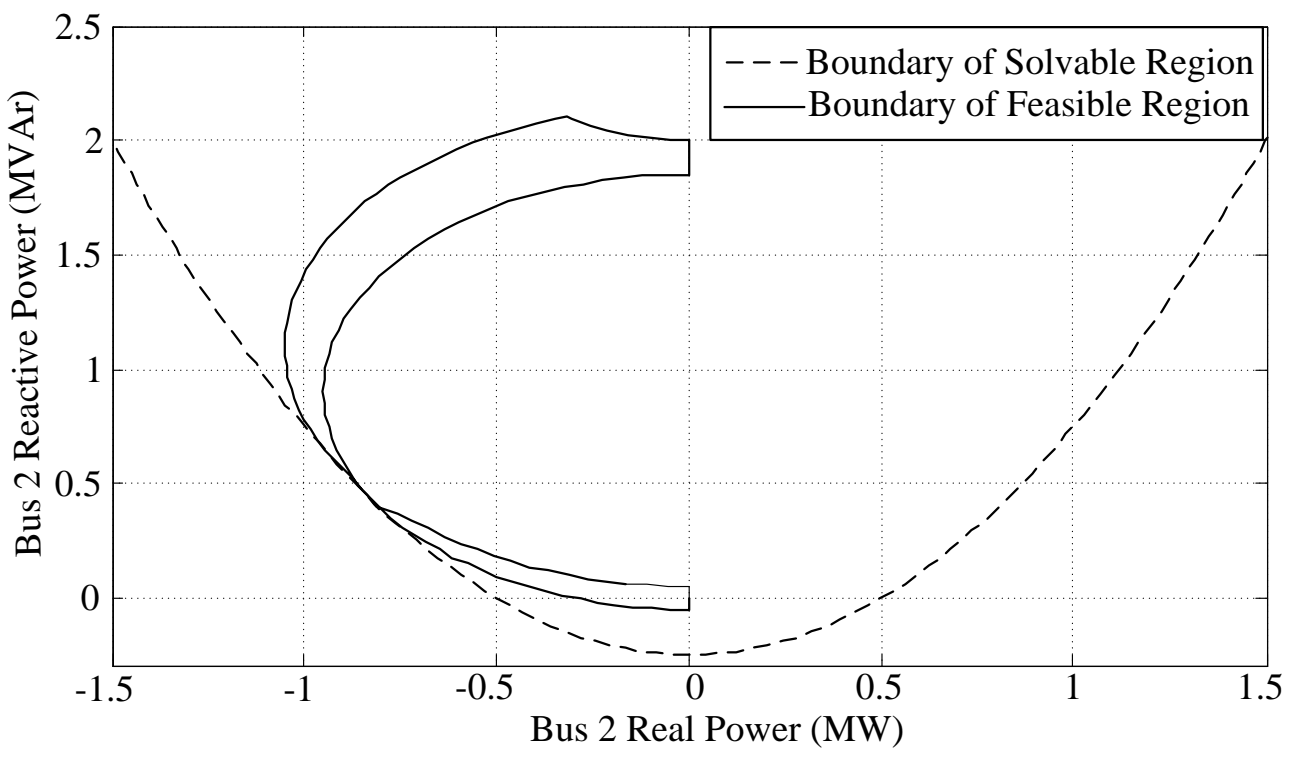

Fig. 10. Feasible region and solvable boundary of the two-bus system [23].

\subsection{Feasible Region with PSO Algorithm}

Table 1. Bus Data [29].

\begin{tabular}{ccccccc}
\hline \multirow{2}{*}{ Bus } & \multicolumn{2}{c}{ Voltage } & \multicolumn{2}{c}{ Generation } & \multicolumn{2}{c}{ Load } \\
& Mag (p.u.) & Ang (deg) & P (MW) & Q (MVAr) & P (MW) & Q (MVAr) \\
\hline 1 & 1.035 & -7.278 & 172.00 & 21.47 & 108.00 & 22.00 \\
2 & 1.035 & -7.370 & 172.00 & 15.66 & 97.00 & 20.00 \\
3 & 0.989 & -5.584 & - & - & 180.00 & 37.00 \\
4 & 0.998 & -9.690 & - & - & 74.00 & 15.00 \\
5 & 1.019 & -9.964 & - & - & 71.00 & 14.00 \\
6 & 1.012 & -12.421 & - & - & 136.00 & 28.00 \\
7 & 1.025 & -7.357 & 240.00 & 51.84 & 125.00 & 25.00 \\
8 & 0.993 & -11.088 & - & - & 171.00 & 35.00 \\
9 & 1.001 & -7.435 & - & - & 175.00 & 36.00 \\
10 & 1.028 & -9.503 & - & - & 195.00 & 40.00 \\
11 & 0.990 & -2.154 & - & - & - & - \\
12 & 1.003 & -1.517 & - & - & - & - \\
13 & 1.020 & 0.000 & 187.25 & 133.99 & 265.00 & 54.00 \\
14 & 0.980 & 2.258 & 0.00 & -27.72 & 194.00 & 39.00 \\
15 & 1.014 & 11.566 & 215.00 & -3.95 & 317.00 & 64.00 \\
16 & 1.017 & 10.449 & 155.00 & 44.40 & 100.00 & 20.00 \\
17 & 1.039 & 14.931 & - & - & - & - \\
18 & 1.050 & 16.292 & 400.00 & 138.73 & 333.00 & 68.00 \\
19 & 1.023 & 8.917 & - & - & 181.00 & 37.00 \\
20 & 1.038 & 9.530 & - & - & 128.00 & 26.00 \\
21 & 1.050 & 17.117 & 400.00 & 106.91 & - & - \\
22 & 1.050 & 22.766 & 300.00 & -29.55 & - & - \\
23 & 1.050 & 10.572 & 660.00 & 135.59 & - & - \\
24 & 0.978 & 5.299 & - & - & - & - \\
& & Total: & 2901.25 & 587.36 & 2850.00 & 580.00 \\
\hline
\end{tabular}


Table 2. Reactance and Capacity Data of Transmission Lines [29].

\begin{tabular}{cccccccc}
\hline From & To & $\begin{array}{c}\text { Reactance } \\
\text { (p.u.) }\end{array}$ & $\begin{array}{c}\text { Capacity } \\
\text { (MVA) }\end{array}$ & From & To & $\begin{array}{c}\text { Reactance } \\
\text { (p.u.) }\end{array}$ & $\begin{array}{c}\text { Capacity } \\
\text { (MVA) }\end{array}$ \\
\hline 1 & 2 & 0.0146 & 175 & 11 & 13 & 0.0488 & 500 \\
1 & 3 & 0.2253 & 175 & 11 & 14 & 0.0426 & 500 \\
1 & 5 & 0.0907 & 350 & 12 & 13 & 0.0488 & 500 \\
2 & 4 & 0.1356 & 175 & 12 & 23 & 0.0985 & 500 \\
2 & 6 & 0.2050 & 175 & 13 & 23 & 0.0884 & 500 \\
3 & 9 & 0.1271 & 175 & 14 & 16 & 0.0594 & 500 \\
3 & 24 & 0.0840 & 400 & 15 & 16 & 0.0172 & 500 \\
4 & 9 & 0.1110 & 175 & 15 & 21 & 0.0249 & 1000 \\
5 & 10 & 0.0940 & 350 & 15 & 24 & 0.0529 & 500 \\
6 & 10 & 0.0642 & 175 & 16 & 17 & 0.0263 & 500 \\
7 & 8 & 0.0652 & 350 & 16 & 19 & 0.0234 & 500 \\
8 & 9 & 0.1762 & 175 & 17 & 18 & 0.0143 & 500 \\
8 & 10 & 0.1762 & 175 & 17 & 22 & 0.1069 & 500 \\
9 & 11 & 0.0840 & 400 & 18 & 21 & 0.0132 & 1000 \\
9 & 12 & 0.0840 & 400 & 19 & 20 & 0.0203 & 1000 \\
10 & 11 & 0.0840 & 400 & 20 & 23 & 0.0112 & 1000 \\
10 & 12 & 0.0840 & 400 & 21 & 22 & 0.0692 & 500 \\
\hline
\end{tabular}

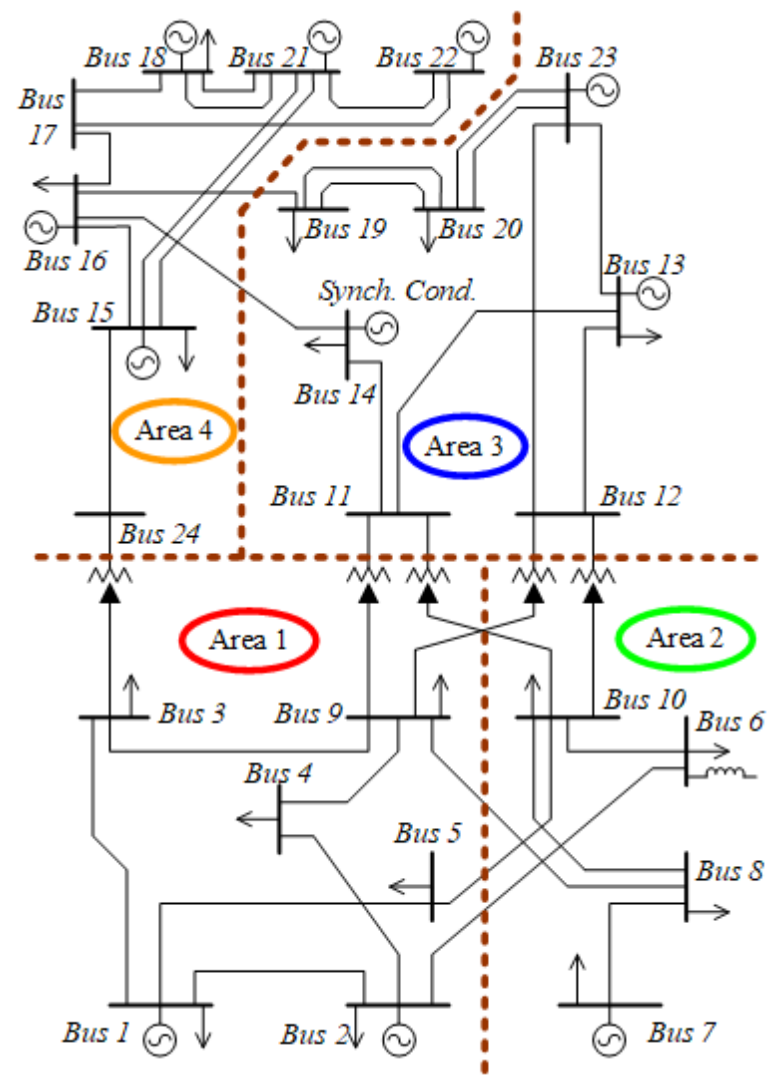

Fig. 11. IEEE 24-bus power system RTS-96 [29]. 


\subsubsection{Result}

Case 1: available transfer capability from area 3 to area 4 taking time 7.8707 seconds.

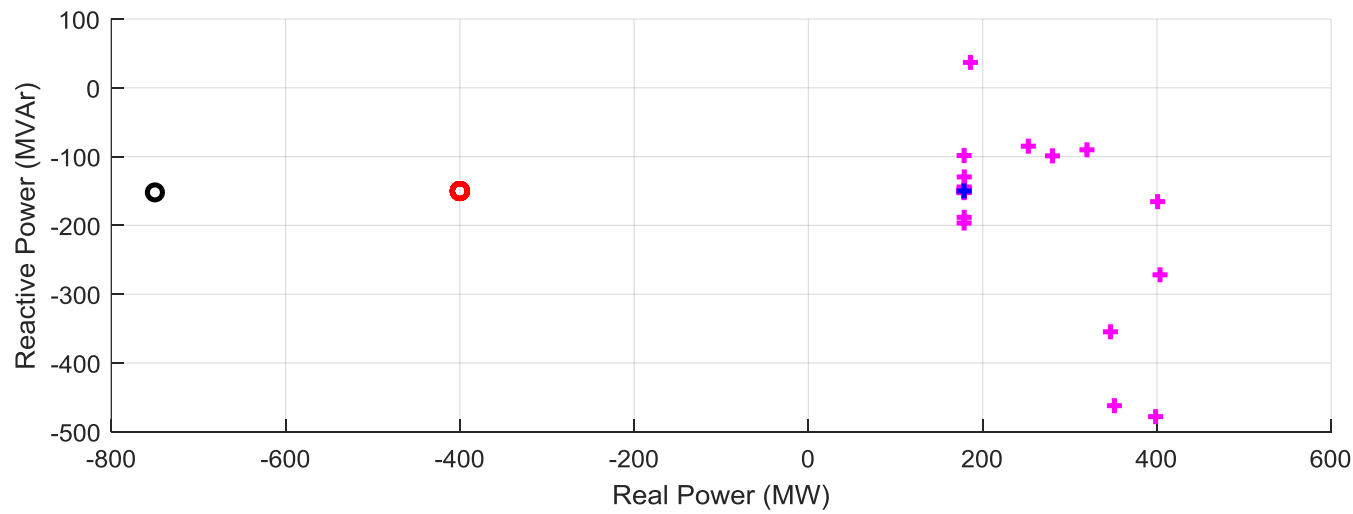

Fig. 12. Tracing boundary method adaptive PSO transfer power from area 3 to area 4.

In the simulation, the red point is the operating load and the black point is the prediction point. The result in the right-hand side of the table shows the available of bus and period of power when the contingency occurs in the power lines system. The blue point is represented as the first initial of process of the particle swarm optimization. From the figure shown the formulation, when the contingency occurs in transmission line, the number of busses that can normally operate is 16 buses. It means that there are the maximum number of buses of 8 buses that can be failed in the system. The period of energy buses is from 170 to $410 \mathrm{MW}$ and $\pm 500 \mathrm{MVAr}$, which is the first operating point at $-400-j 150 \mathrm{MVAr}$ as the point inside load feasible region. In addition, the initial point of tracing boundary method is $-780-j 150 \mathrm{MVA}$, which is the first point to predict the transformation of the load.

Case 2: available transfer capability from area 2 to area 4 taking time 5.7323 seconds.

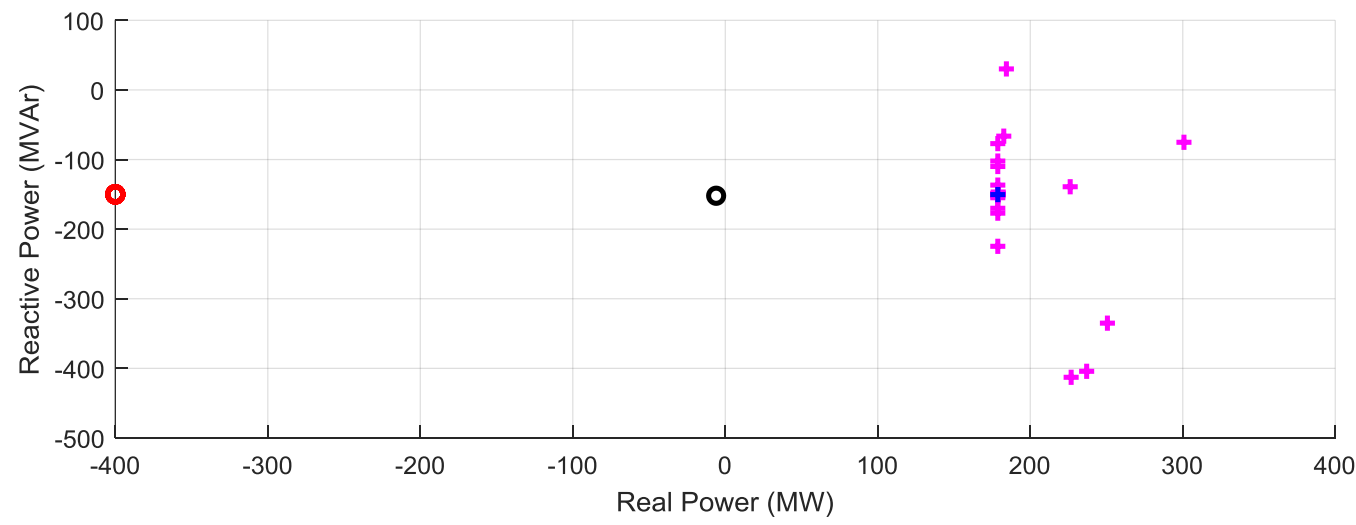

Fig. 13. Tracing boundary method adaptive PSO transfer power from area 2 to area 4.

From the figure, it shows the formulation when the contingency occurs in transmission line. The number of buses that can normally operate are 17 buses. It means that there are the maximum number of buses of 7 buses that can be failed in the system. The period of energy buses is from 180 to $300 \mathrm{MW}$ and $\pm 420 \mathrm{MVAr}$, which is the first operating point at $-400-j 150 \mathrm{MVAr}$ as the point inside load feasible region. In addition, the initial point of tracing boundary method is $-5-j 150 \mathrm{MVA}$, which is the first point to predict the transformation of the load. 
Case 3: available transfer capability from area 1 to area 4 taking time 5.5689 seconds.

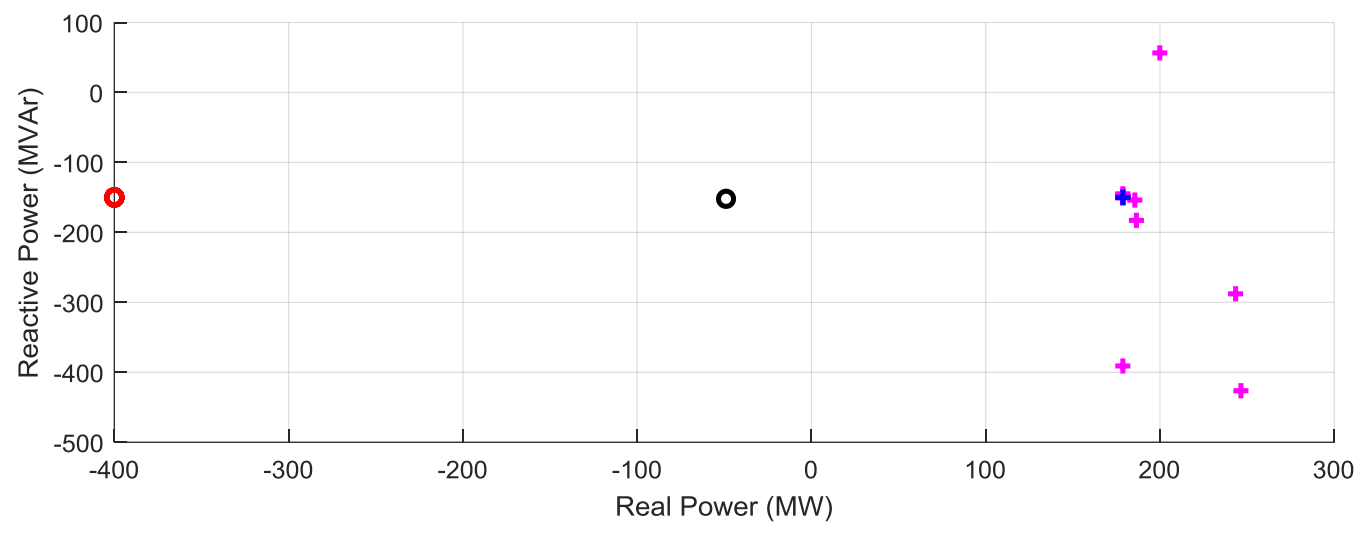

Fig. 14. Tracing boundary method adaptive PSO transfer power from area 1 to area 4 .

From the figure, it shows the formulation when the contingency occurs in transmission line. The number of buses that can normally operate are 8 buses; however, there are the maximum number of buses of 16 buses that can be failed in the system. In this case, it is impossible. The period of energy buses is from 180 to 250 MW and $\pm 420 \mathrm{MVAr}$, which is the first operating point at -400-j150 MVAr as the point inside load feasible region. In addition, the initial point of tracing boundary method is -50-j150 MVA, which is the first point to predict the transformation of the load. On the other hand, it is possible that the result of the particle swarm is outside the solvable boundary because the area 1 insufficiently generates power to meet load demand in the area 4.

Case 4: available transfer capability from area 4 to area 3 taking time 5.2816 seconds.

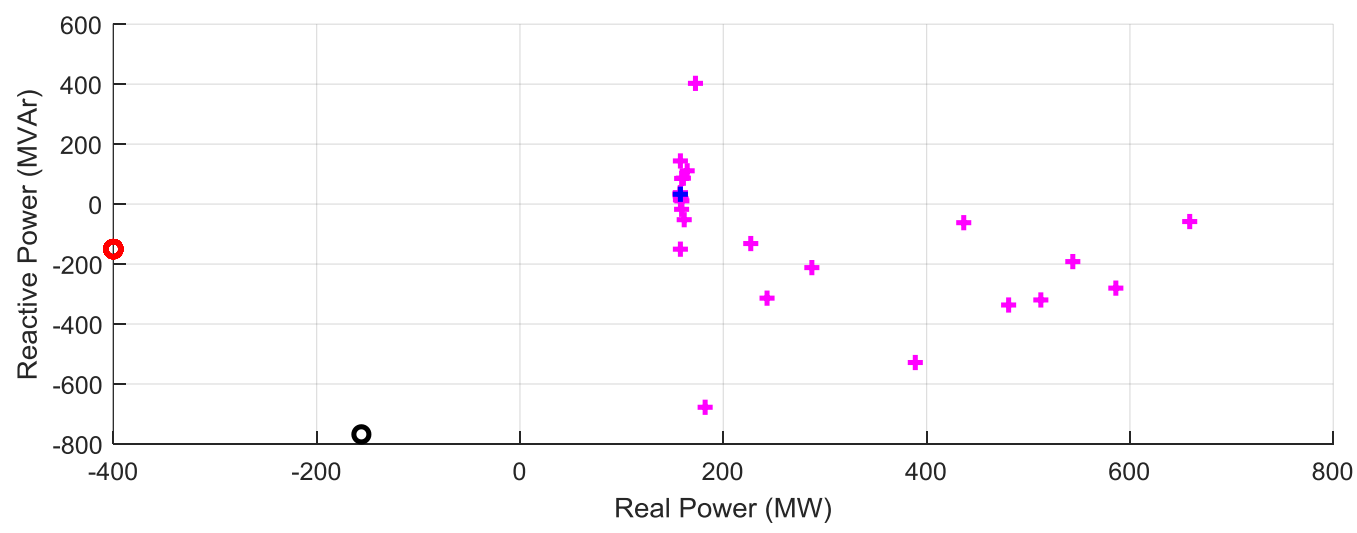

Fig. 15. Tracing boundary method adaptive PSO transfer power from area 4 to area 3.

From the figure, it shows the formulation when the contingency occurs in transmission line. The number of buses that can normally operate are 20 buses. It means that there are the maximum number of buses of 4 buses that can be failed in the system. The period of energy buses is from 180 to $640 \mathrm{MW}$ and $\pm 650 \mathrm{MVAr}$, which is the first operating point at $-400-j 180 \mathrm{MVAr}$ as the point inside load feasible region. In addition, the initial point of tracing boundary method is $-180-j 790 \mathrm{MVA}$, which is the first point to predict the transformation of the load. 
Case 5: available transfer capability from area 2 to area 3 taking time 6.0676 seconds.

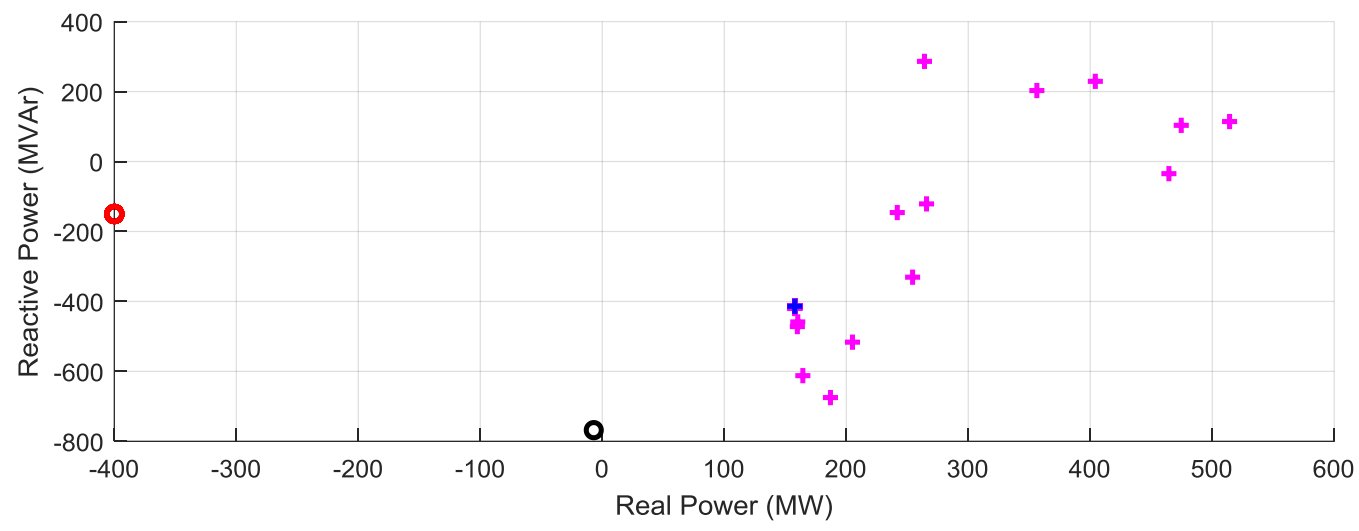

Fig. 16. Tracing boundary method adaptive PSO transfer power from area 2 to area 3.

From the figure, it shows the formulation when the contingency occurs in transmission line. The number of buses that can normally operate are 16 buses. It means that there are the maximum number of buses of 8 buses that can be failed in the system. The period of energy buses is from 150 to $530 \mathrm{MW}$ and $\pm 700 \mathrm{MVAr}$ because of this case there are several load levels, consequently the period of load that it varies. The first operating point is - $400-j 180 \mathrm{MVAr}$ as the point inside load feasible region. In addition, the initial point of tracing boundary method is $-10-j 790 \mathrm{MVA}$, which is the first point to predict the transformation of the load.

Case 6: available transfer capability from area 1 to area 3 taking time 5.5012 seconds.

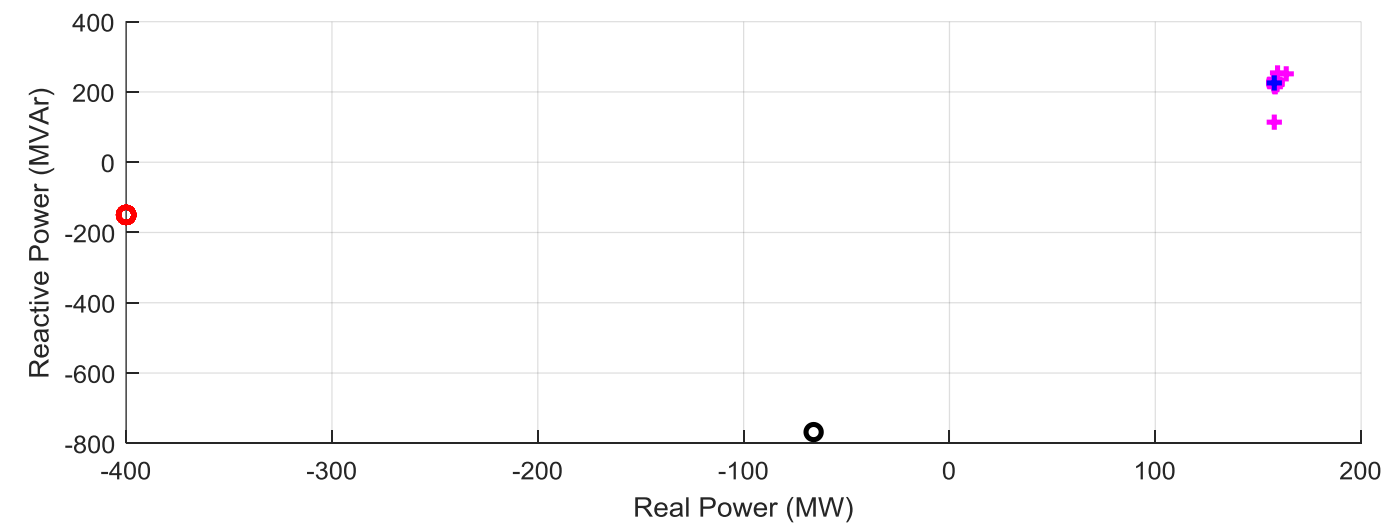

Fig. 17. Tracing boundary method adaptive PSO transfer power from area 1 to area 3.

From the figure, it shows the formulation when the contingency occurs in transmission line. The number of buses that can normally operate are 5 buses; however, there are the maximum number of buses of 19 buses that can be failed in the system. In this case, it is impossible. The period of energy buses is from 150 to 160 MW and $\pm 250 \mathrm{MVAr}$, which is the first operating point at -400-j180 MVAr as the point inside load feasible region. In addition, the initial point of tracing boundary method is -60-j790 MVA, which is the first point to predict the transformation of the load. On the other hand, it is possible that the result of the particle swarm is outside the solvable boundary because the area 1 insufficiently generates power to meet load demand in the area 3. 
Case 7: available transfer capability from area 4 to area 2 taking time 5.6305 seconds.

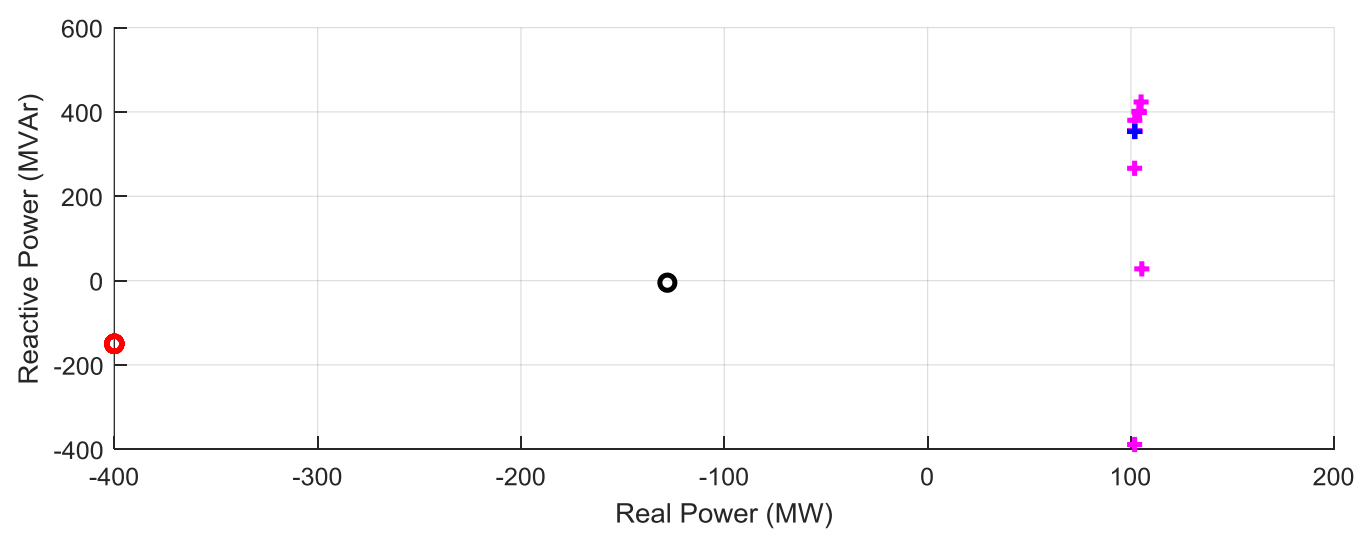

Fig. 18. Tracing boundary method adaptive PSO transfer power from area 4 to area 2 .

From the figure, it shows the formulation when the contingency occurs in transmission line. The number of buses that can normally operate are 9 buses; however, there are the maximum number of buses of 15 buses that can be failed in the system. In this case, it is impossible. The period of energy buses is from 100 to 110 MW and \pm 250 MVAr, therefore not different load level because of area two may be less loaded or the load consumption is similarly and consistent. The first operating point at $-400-j 180 \mathrm{MVAr}$ as the point inside load feasible region. In addition, the initial point of tracing boundary method is -40-j0 MVA, which is the first point to predict the transformation of the load. On the other hand, it is possible that the result of the particle swarm is outside the solvable boundary because the area 4 insufficiently generates power to meet load demand in the area 2.

Case 8: available transfer capability from area 3 to area 2 taking time 5.6298 seconds.

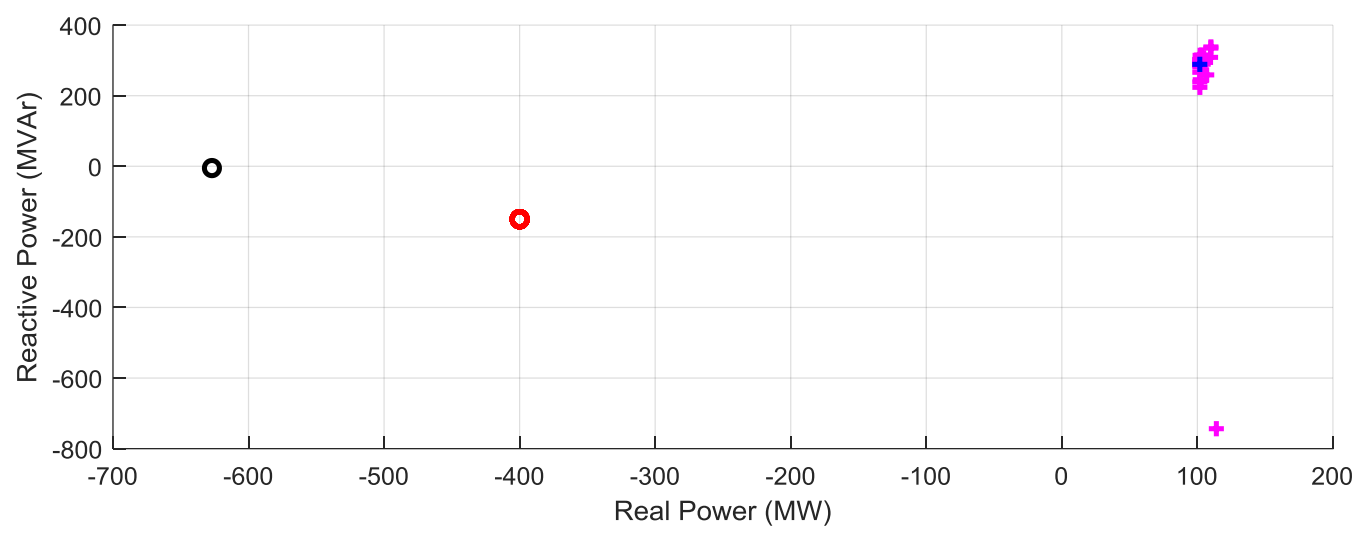

Fig. 19. Tracing boundary method adaptive PSO transfer power from area 3 to area 2.

From the figure, it shows the formulation when the contingency occurs in transmission line. The number of buses that can normally operate are 9 buses; however; there are the maximum number of buses of 15 buses that can be failed in the system. In this case, it is impossible. The period of energy buses is from 100 to 110 MW and \pm 250 MVAr, therefore not different load level because of area two may be less loaded or the load consumption is similarly and consistent. The first operating point at $-400-j 180 \mathrm{MVAr}$ as the point inside load feasible region. In addition, the initial point of tracing boundary method is -40-j0 MVA, which is the first point to predict the transformation of the load. On the other hand, it is possible that the result of the particle swarm is outside the solvable boundary because the area 3 insufficiently generates power to meet load demand in the area 2. 
Case 9: available transfer capability from area 1 to area 2 taking time 5.6598 seconds.

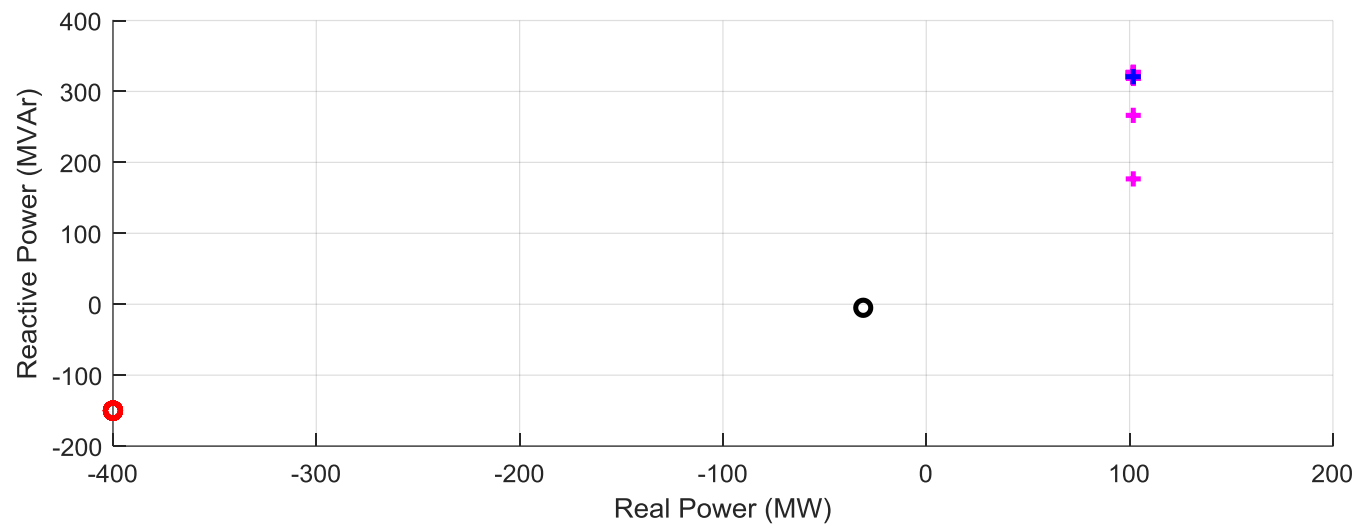

Fig. 20. Tracing boundary method adaptive PSO transfer power from area 1 to area 2.

From the figure, it shows the formulation when the contingency occurs in transmission line. The number of buses that can normally operate are 5 buses; however, there are the maximum number of buses of 19 buses that can be failed in the system. In this case, it is impossible. The period of energy buses is from 100 to 110 MW and $\pm 250 \mathrm{MVAr}$, therefore not different load level because of area two may be less loaded or the load consumption is similarly and consistent. The first operating point at $-400-j 150 \mathrm{MVAr}$ as the point inside load feasible region. In addition, the initial point of tracing boundary method is -40-j0 MVA, which is the first point to predict the transformation of the load. On the other hand, it is possible that the result of the particle swarm is outside the solvable boundary because the area 1 and area 2 contain less the number of buses than the other areas. Consequently, they affect the parameters.

Case 10: available transfer capability from area 4 to area 1 taking time 5.4238 seconds.

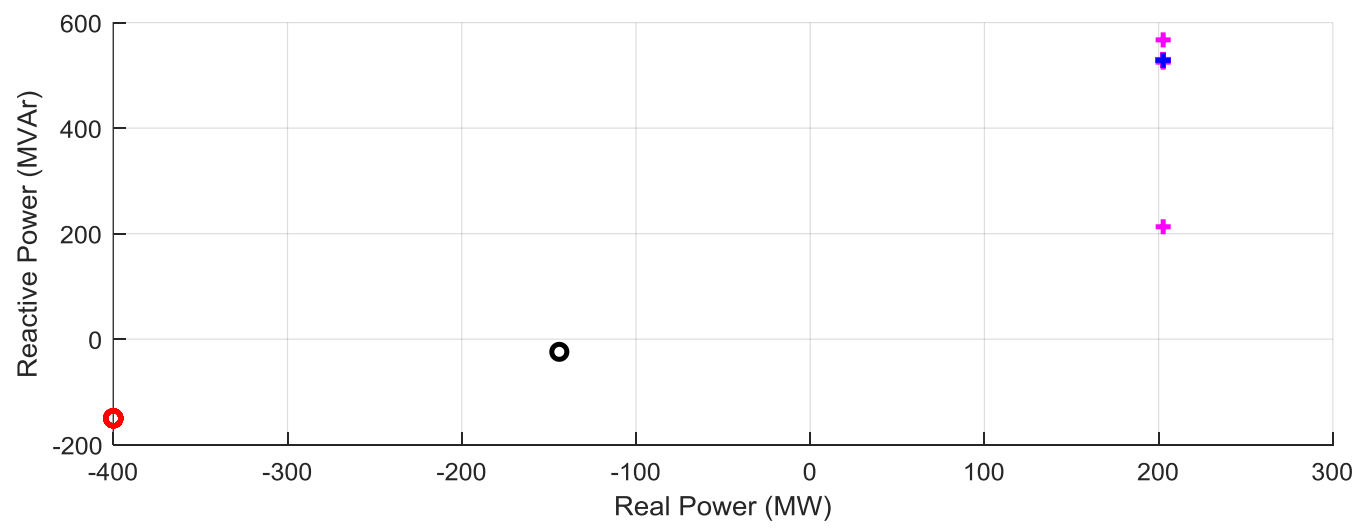

Fig. 21. Tracing boundary method adaptive PSO transfer power from area 4 to area 1.

From the figure, it shows the formulation when the contingency occurs in transmission line. The number of buses that can normally operate are 5 buses; however, there are the maximum number of buses of 19 buses that can be failed in the system. In this case, it is impossible. The period of energy buses is from 100 to 110 MW and \pm 250 MVAr, therefore not different load level because of area two may be less loaded or the load consumption is similarly and consistent. The first operating point at $-400-j 180 \mathrm{MVAr}$ as the point inside load feasible region. In addition, the initial point of tracing boundary method is -40-j0 MVA, which is the first point to predict the transformation of the load. On the other hand, it is possible that the result of the particle swarm is outside the solvable boundary because the area 1 insufficiently generates power to meet load demand in the area 4. 
Case 11: available transfer capability from area 3 to area 1 taking time 5.7610 seconds.

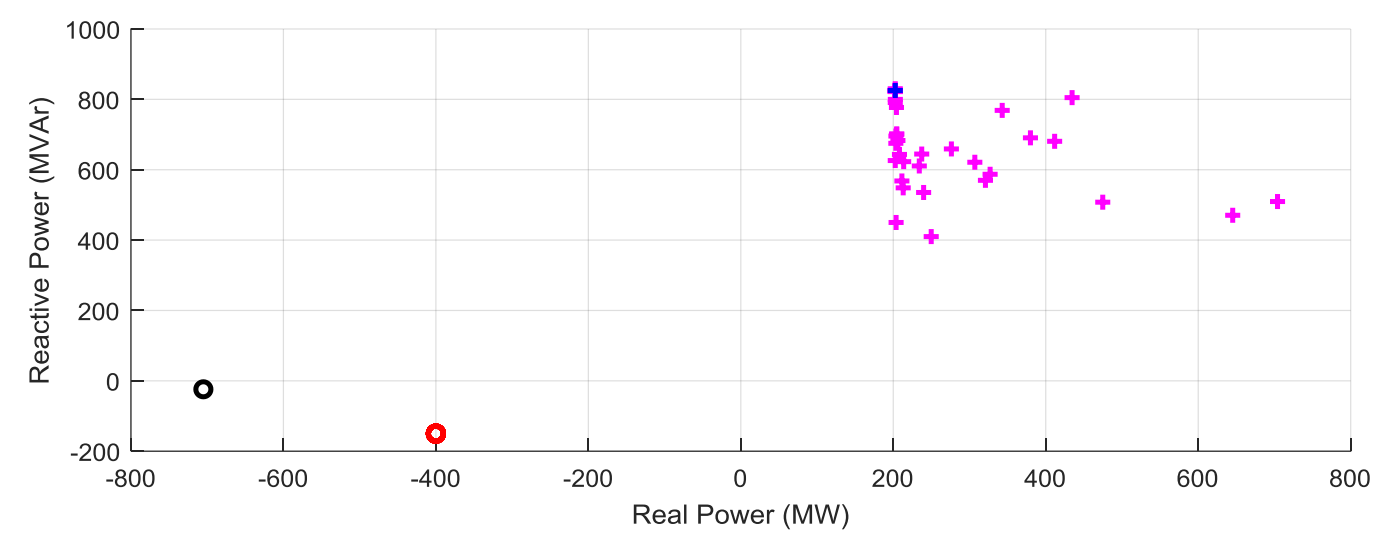

Fig. 22. Tracing boundary method adaptive PSO transfer power from area 3 to area 1.

From the figure, it shows the formulation when the contingency occurs in transmission line. The number of buses that can normally operate are 22 buses. It means that there are the maximum number of buses of 2 buses that can be failed in the system. The period of energy buses is from 100 to $110 \mathrm{MW}$ and $\pm 250 \mathrm{MVAr}$ because of the system has a different load level. The first operating point at -400-j180 MVAr as the point inside load feasible region. Therefore, the initial point of tracing boundary method is - $700-j 30 \mathrm{MVA}$, which is the first point to predict the transformation of the load. In addition, it is possible that the system is quite stable due to the replacement of a lot of loads. The production of energy in the area 3 is sufficient for load demands, in case that the generator in area 1 cannot operate.

Case 12: available transfer capability from area 2 to area 1 taking time 5.8216 seconds.

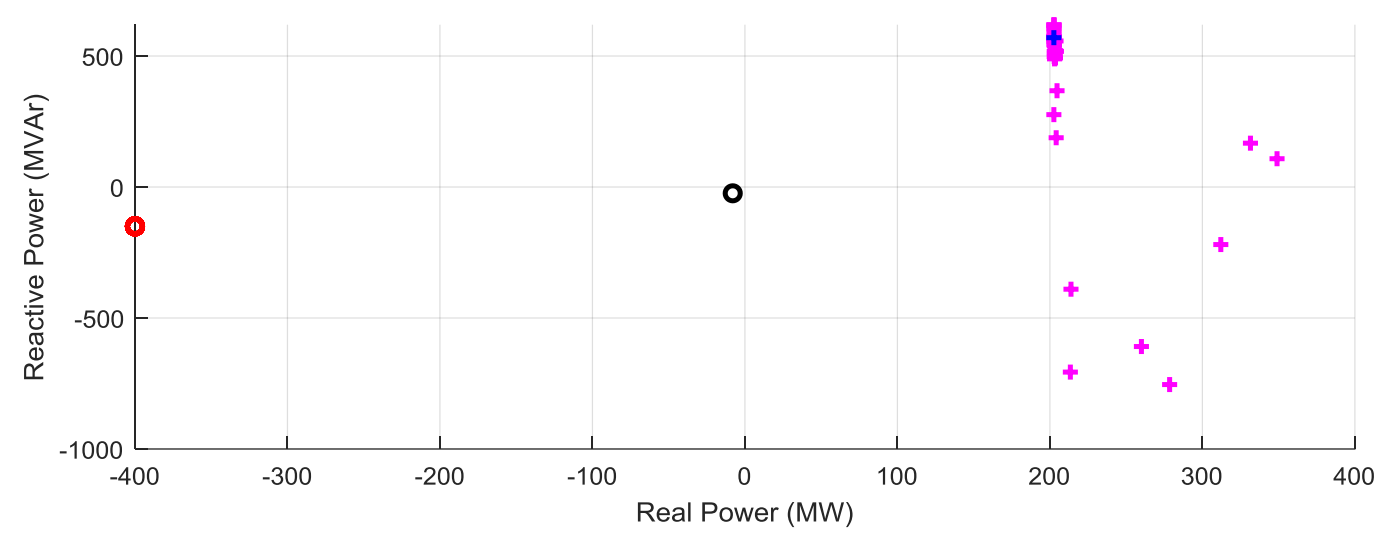

Fig. 23. Tracing boundary method adaptive PSO transfer power from area 2 to area 1.

From the figure, it shows the formulation when the contingency occurs in transmission line. The number of buses that can normally operate are 16 buses. It means that there are the maximum number of buses of 8 buses that can be failed in the system. The period of energy buses is from 200 to $350 \mathrm{MW}$ and $\pm 700 \mathrm{MVAr}$ because of the system has a different load level. The first operating point at $-400-j 180$ MVAr as the point inside load feasible region. In addition, the initial point of tracing boundary method is -10-j30 MVA, which is the first point to predict the transformation of the load. 


\section{Conclusions}

The boundary of feasible region and particle swarm are important for determining the robustness of operating points. The feasible region is the set of the power flow solutions without any operational violation, and particle swarm is the set of the groups that can find the most appropriate result in the event of a problem with multiple answers. This paper proposes a method for tracing the feasible boundary adaptive with particle swarm of the considered load bus. The optimization-based method is able to solve the feasible boundary point and control power injection by assigning free variables and minimizing operational point limits. The modified predictorcorrector process can trace the feasible boundary to obtain the complete contour of feasible boundary, and the particle swarm optimization can trace boundary point for controlling the injection in the two-dimensional space.

Table 3. The number of results of IEEE 24-bus test system.

\begin{tabular}{ccccccccc}
\hline Case & $\begin{array}{c}\text { Source } \\
\text { Area }\end{array}$ & $\begin{array}{c}\text { Source } \\
\text { Area }\end{array}$ & $\begin{array}{c}\text { Maximum } \\
\text { Load } \\
\text { (MW) }\end{array}$ & $\begin{array}{c}\text { Minimum } \\
\text { Load } \\
\text { (MW) }\end{array}$ & $\begin{array}{c}\text { Times } \\
\text { (Second) }\end{array}$ & $\begin{array}{c}\text { Number } \\
\text { of bus } \\
\text { operation }\end{array}$ & $\begin{array}{c}\text { Initial } \\
\text { prediction } \\
\text { point (MVA) }\end{array}$ & $\begin{array}{c}\text { Time } \\
\text { per } \\
\text { point }\end{array}$ \\
\hline 1 & 3 & 4 & 410 & 170 & 7.8707 & 16 & $-400-j 150$ & 0.4919 \\
2 & 2 & 4 & 300 & 180 & 5.7323 & 17 & $-400-j 150$ & 0.3371 \\
3 & 1 & 4 & 250 & 180 & 5.5689 & $8^{*}$ & $-400-j 150$ & 0.6961 \\
4 & 4 & 3 & 640 & 180 & 5.2816 & 22 & $-400-j 180$ & 0.2400 \\
5 & 2 & 3 & 530 & 150 & 6.0675 & 16 & $-400-j 180$ & 0.3792 \\
6 & 1 & 3 & 160 & 150 & 5.5012 & $5^{*}$ & $-400-j 180$ & 1.1002 \\
7 & 4 & 2 & 110 & 100 & 5.6304 & $9 *$ & $-400-j 180$ & 0.6256 \\
8 & 3 & 2 & 110 & 100 & 5.6297 & $9 *$ & $-400-j 180$ & 0.6255 \\
9 & 1 & 2 & 105 & 100 & 5.6597 & $5^{*}$ & $-400-j 150$ & 1.1319 \\
10 & 4 & 1 & 105 & 100 & 5.4238 & $5^{*}$ & $-400-j 180$ & 0.6779 \\
11 & 3 & 1 & 650 & 200 & 5.7610 & 22 & $-400-j 180$ & 0.2618 \\
12 & 2 & 1 & 350 & 200 & 5.8216 & 16 & $-400-j 180$ & 0.3638 \\
\hline
\end{tabular}

From the table, it can be noted that there is still a total of 6 areas of the system, from the next 6 areas, as the other 2 areas of the particle swarm cannot be randomly searched because they are outside the feasible region or solvable region as the known area where the value cannot be found as a result of the parameters used for incomplete analysis, for example, the source area and the load area are not corresponding to the demand energy therefore the source area may not be able to produce as much as the demand for energy or the side of the delivery of the load area. In addition, there are other factors to be involved, for example, some case can be the solution, but the answer range is in an area where both of the values can be determined and cannot be determined, which particle swarm is not reached the result.

From the numerical examples, the results demonstrate that the feasible region visualization enhances the security monitoring. With the considered operating load, the region shows the load margins in many directions including the limitation of control actions, e.g. the available reactive power compensated by capacitor banks and the available load shedding. Moreover, the different assignment of free variables enables the method to find the several types of feasible region point to control power injection. The proposed method is also able to trace the feasible boundary point when generator voltages are free; in order to observe the robustness of loading points and optimal value of active power and reactive power for compensation. Furthermore, when the loads of other buses as free variables are defined, the obtained result is the outermost boundary that shows the potential of the system to supply the considered bus. 


\section{References}

[1] V. Ajjarapu and C. Christy, "The continuation power flow: a tool for steady state voltage stability analysis," IEEE Transaction on Power Systems, vol. 7, no. 1, pp. 416-423, Feb. 1992.

[2] H. D. Chiang, A. J. Flueck, K. S. Shah, and N. Balu, "CPFLOW: A practical tool for tracing power system steady-state stationary behavior due to load and generation variations," IEEE Transactions on Power Systems, vol. 10, no. 2, pp. 623-634, May 1995.

[3] NERC. (1996). Available Transfer Capability Definitions and Determination [Online]. Available: http://www.nerc.com/ filez/atcwg.html [Accessed: 17 June 2006]

[4] B. A. Khairuddin, S. S. Ahmed, W. M. Mustafa, A. A. Zin, and H. Ahmad "A novel method for ATC computations in a large-scale power system," TEEE Transaction on Power Systems, vol. 19, no. 2, pp. 1150-1158, May 2004.

[5] Y. Ou and C. Sigh, "Assessment of available transfer capability and margin," IEEE Transaction on Power Systems, vol. 17, no. 2, pp. 463-468, May 2002.

[6] W. Li and P. Wang, "Determination of optimal total transfer capability using a probabilistic approach," IEEE Transaction on Power Systems, vol. 21, no. 2, pp. 862-868, May. 2006.

[7] L. Min and A. Abur, "Total transfer capability computation for multi-area power system," IEEE Transaction on Power Systems, vol. 21, no. 3, pp. 1141-1147, Aug. 2006.

[8] R. M. Alrashidi and E. M. El-Hawary, "A survey of particle swarm optimization applications in electric power systems," IEEE Transaction on Evolutionary Computation, vol. 13, no. 1, pp. 913-918, Aug. 2009.

[9] A. Verma, B. K. Panigrahi, and P. R. Bijwe, "Transmission network expansion planning with adaptive particle swarm optimization," in 2009 World Congress on Nature and Biologically Inspired Computing (NaBIC), IEEE, 2009, pp. 1099-1104.

[10] J. T. Overbye, "A power flow measure for unsolvable cases," IEEE Transaction on Power Systems, vol. 9, no. 3, pp. 1359-1365, Aug. 1994.

[11] J. T. Overbye, "Computation of a practical method to restore power flow solvability," IEEE Transaction on Power Systems, vol. 10, no. 1, pp. 280-287, Feb. 1995.

[12] S. N. Singh and S. C. Srivastava, "Corrective action planning to achieve a feasible optimal power flow solution," IEE Proceeding Generation Transmission and Distribution, vol. 142, no. 6, pp. 576-582, Nov. 1995.

[13] S. Granville, O. C. J. Mello, and G. C. A. Melo, "Application of interior point methods to power flow unsolvability," IEEE Transaction on Power Systems, vol. 11, no. 2, pp. 1096-1103, May 1996.

[14] C. G. A. Conceicao and A. C. Castro, "A new approach to defining corrective control actions in case of infeasible operating situations," in IEEE Porto Power Tech Conference, Sep, 2001, vol. 3.

[15] A. I. Hiskens and J. R. Davy, "Exploring the power flow solution space boundary," IEEE Transaction on Power Systems, vol. 16, no. 3, pp. 389-395, Aug. 2001.

[16] A. C. Canizares and L. F. Avarado, "Point of collapse and continuation methods for large AC/DC systems," IEEE Transaction on Power Systems, vol. 8, no. 1, pp. 1-8, Feb. 1993.

[17] V. Y. Makarov, J. D. Hill, and Y. Z. Dong, "Computation of bifuecation boundaries for power systems: A new $\Delta$-plane method," IEEE Transaction on Circuits and Systems, vol. 47, no. 4, pp. 536-544, Apr. 2000.

[18] V. Ajjarapu, Computational Techniques for Voltage Stability Assessment and Control. New York: Springer, 2006.

[19] Y. Yu, P. Li, H. Jia, T. S. Lee, and P. Zhang, "Computation of boundary of power flow feasible region with hybrid method," in Proceedings of Power Systems Conference and Exposition, IEEE PES, New York, Oct. 2004, vol. 1, pp. 137-143.

[20] S. Limpatthamapanee and S. Phichaisawat, "Visualization of load security region boundary by operational constraints of power systems," Engineering Journal, vol. 15, no. 3, pp. 1-73, Jul. 2011.

[21] V. Ajjarapu and C. Christy, "The continuation power flow: A tool for steady state voltage stability analysis," IEEE Transaction on Power Systems, vol. 7, no. 1, pp. 416-423, Feb. 1992.

[22] S. Limpatthamapanee and S. Phichaisawat, "Determination of transfer capability region using boundary tracing method," IEEJ Transactions on Electrical and Electronic Engineering, vol. 7, pp. 375-382, Jun. 2011.

[23] S. Limpatthamapanee, "Load feasible region determination using boundary tracing method," Ph.D. dissertation, Department of Electrical Engineering, Chulalongkorn University, Pathumwan, Bangkok, 2011.

[24] S. Auchariyamet, "Optimal planning and operation of reactive power management in distribution system by particle swarm optimization," Ph.D. dissertation, Department of Electrical Engineering, King Mongkut's University of Technology North Bangkok, Bangsue, Bangkok, Thailand, 2010. 
[25] R. C. Eberhart and Y. Shi, "Comparison between genetic algorithms and particle swarm optimization," in Proc. IEEE Int. Conf. Evol. Comput., May, 1998, pp. 611-616.

[26] R. C. Eberhart and Y. Shi, "Particle swarm optimization: Development, applications and resources, in Proc. Congress on Evolutionary Computation, Soul, 2001, pp. 81-86.

[27] Y. Shi and R. C. Eberhart, "Fuzzy adaptive particle swarm optimization," in Proceedings of Evolutionary Computation, 27-30 May 2001, vol. 1, pp 101-106.

[28] S. Auchariyamet and S. Sirisumrannukul, "Optimal allocation of static Var compensator for active power loss reduction by different decision variables," GMSARN International Journal, vol. 4, no. 2, pp. 57-66, Jun. 2010.

[29] C. Grigg, P. Wong, P. Albrecht, R. Allan, M. Bhavaraju, R. Billinton, Q. Chen, C. Fong, S. Haddad, S. Kuruganty, and W. Li, "Reliability test system task force:'The IEEE reliability test system 1996'," IEEE Trans Power Syst, vol. 14, no. 3, pp.1010-1020, 1999. 